\title{
Regionalización de sequías a partir del SPI en la Demarcación Hidrográfica del Segura y Mancomunidad de los Canales del Taibilla (sureste de España)
}

Regionalization of droughts from the SPI in the Segura hydrographic basin and Commonwealth of Taibilla Channels (Southeast of Spain)

\author{
Víctor Ruiz Álvarez \\ victor.ruiz1@um.es \\ Departamento de Geografía \\ Universidad de Murcia (España) \\ María Manuela Portela \\ maria.manuela.portela@ist.utl.pt \\ Instituto Superior Técnico \\ Universidad de Lisboa (Portugal) \\ Luis Ángel Espinosa \\ luis.espinosa@tecnico.ulisboa.pt \\ Instituto Superior Técnico \\ Universidad de Lisboa (Portugal)
}




\section{Ramón García Marín}

ramongm@um.es

Departamento de Geografía

Universidad de Murcia (España)

\section{Francisco Belmonte Serrato}

franbel@um.es

Departamento de Geografía

Universidad de Murcia (España)

\section{Marcos Ruiz Álvarez}

marcos.ruiz1@um.es

Departamento de Geografía

Universidad de Murcia (España)

\section{Resumen}

A partir de un total de 92 series de precipitación mensual de la base de datos de la Agencia Estatal de Meteorología (AEMET) se ha realizado un análisis exhaustivo de sequías en el marco espacial de la Demarcación Hidrográfica del Segura (DHS) y Mancomunidad de los Canales del Taibilla (MCT), organismos encargados de la planificación de los recursos hídricos, y del abastecimiento de agua potable, respectivamente, de gran parte del sureste de España. El objetivo principal de este estudio es la regionalización de los patrones temporales de sequía a partir del Standarized Precipitation Index (SPI), con escala de 12 meses (SPI12), para evaluar las sequías hidrológicas. El periodo seleccionado es 1968-2017. La regionalización se ha realizado a partir del Análisis de Componentes Principales (ACP), a través del cual se han obtenido 4 regiones homogéneas. A partir de estas regiones se ha identificado el porcentaje de área afectada por sequías de diferente severidad, y la frecuencia de ocurrencia de sequías (basado en el método Kernel Ocurrence Rate Estimation-KORE). Se observa un incremento de la frecuencia de ocurrencia de sequías y del área afectada por las mismas en prácticamente todas las regiones, lo que conlleva importantes repercusiones territoriales, sociales y económicas en un ámbito territorial semiárido que presenta un déficit hídrico destacado.

Palabras clave: SPI; análisis componentes principales; sequía; KORE. 


\begin{abstract}
Based on a total of 92 monthly rainfall series from the database of the State Meteorological Agency (AEMET), an exhaustive analysis of droughts has been carried out within the spatial framework of the Segura Hydrographic Basin (DHS) and the Commonwealth of Taibilla Channels (MCT), bodies responsible for planning water resources and the supply of drinking water, respectively, for a large part of south-eastern Spain. The main objective of this study is the regionalization of the temporal patterns of drought from the Standardized Precipitation Index (SPI), with a 12-month scale (SPI12), to evaluate the hydrological droughts. The selected period is 1968-2017. The regionalization has been carried out from the Principal Component Analysis (PCA), through which 4 homogeneous regions have been obtained. From these regions, the percentage of area affected by droughts of different severity and the frequency of occurrence of droughts (based on the Kernel Ocurrence Rate Estimation-KORE method) have been identified. An increase in the frequency of occurrence of droughts and in the area affected by them is observed in practically all regions, which entails important territorial, social and economic repercussions in a semi-arid territorial environment that presents a significant water deficit.
\end{abstract}

Key words: SPI; principal component analysis; drought; KORE.

\title{
1 Introducción
}

A diferencia de otros riesgos naturales como los terremotos o inundaciones que se manifiestan de forma repentina y brusca, las sequías se desarrollan de forma lenta e imperceptible para la población, pudiendo pasar inadvertidas durante mucho tiempo (Tallaksen y Van Lanen, 2004). No obstante, en los últimos años se ha introducido el concepto de "sequía repentina" para referirse a los eventos que presentan un desarrollo muy rápido (Ford et al., 2015; Noguera et al., 2020). En la mayor parte de las situaciones, las sequías constituyen un riesgo silencioso, siendo su manifestación progresiva y constante. Conforme avanza el tiempo sus efectos se van agravando de forma continua hasta que se den las condiciones atmosféricas idóneas para sufragar el déficit pluviométrico acumulado (Olcina, 2006). En las últimas décadas, con respecto a la consideración del riesgo de sequía, y conforme ha ido aumentando el nivel de vida, las sociedades desarrolladas han pasado de la austeridad en el gasto de agua al despilfarro y de la adaptación al catastrofismo (Morales et al., 1999), lo cual aumenta más si cabe la vulnerabilidad de la población frente a las sequías. 
Las sequías son el riesgo natural que mayor número de afectados y repercusiones económicas generan en los territorios donde se desarrolla, como por ejemplo, el ámbito territorial analizado en este trabajo (sureste de España). De hecho, en la clasificación de los riesgos naturales según su vulnerabilidad en el mundo (clasificación obtenida a partir del análisis de las mayores catástrofes naturales ocurridas en la superficie terrestre entre 1900 y 2005), las sequías aparecen en primer lugar según población afectada y en segundo lugar por víctimas causadas, tan solo por detrás de las inundaciones (Olcina, 2006).

Paradójicamente, entre el conjunto de riesgos naturales de origen climático que suponen un riesgo para la actividad humana, la sequía es el que menos atención recibe en los trabajos de investigación, a pesar de ocasionar numerosas pérdidas económicas. De este modo, la falta de consecuencias tangibles a corto plazo y el alivio temporal que producen los episodios de lluvias torrenciales que suelen intercalarse durante los periodos secos en el caso del sureste de España (Olcina, 2001a), provocan entre las administraciones y la población un desinterés que no hace sino agravar los efectos del déficit hídrico consecuente (García-Marín, 2008). En este sentido, cabe destacar la estrecha relación existente entre la gestión de la sequía y la gestión de los recursos hídricos. Si la integración de las estrategias de gestión del riesgo de sequía y de los recursos hídricos resulta insatisfactoria, aumenta de forma considerable la vulnerabilidad de sequía (Vargas \& Paneque, 2019).

Por otra parte, desde un punto de vista geográfico la caracterización de las sequías debe llevarse a cabo teniendo en cuenta sus causas físicas y humanas. En este sentido, en relación con la evaluación del riesgo de sequía, se establecieron los denominados "umbrales de sequía" (Charre, 1977). Este autor expone que el umbral de sequía se sitúa sobre la curva de distribución de la precipitación, aunque son las características sociales, territoriales, económicas e incluso políticas las que determinan finalmente su posición concreta. Wilhite y Glantz (1985) propusieron una clasificación con 4 tipos de sequía (meteorológica, agrícola, hidrológica y socioeconómica), cuyos efectos negativos se van sucediendo de forma secuencial. En la actualidad son los aspectos humanos los que tienen un mayor peso en la valoración de este fenómeno natural, hasta el punto de motivar su propia aparición debido a que las demandas de agua urbana, agraria, hidroeléctrica e industrial han provocado una alteración del umbral de sequía en numerosos ámbitos geográficos como el sureste de España (Olcina, 2001b; Morote, 2019). 
Por tanto, el umbral de sequía evoluciona con la sociedad, y su evolución dependerá tanto de la variación en la distribución temporal y espacial de las precipitaciones, como de los cambios en la capacidad de flexibilización de la oferta y demanda, de los usos del suelo, de la gestión de los estados de sequía y el ritmo de las demandas de agua (Pérez-Cueva, 1983; García-Marín, 2008). En definitiva, para el análisis de las sequías resulta fundamental conocer el estado de la vulnerabilidad de un territorio determinado en la que intervienen múltiples factores humanos y físicos (Del Moral et al., 2017).

Los índices de sequía pluviométrica se constituyen como herramientas imprescindibles para la identificación precisa de los eventos de sequía, pudiendo ayudar en la monitorización y predicción de las mismas (Peña et al., 2016). Desde la década de 1960 se han implementado diferentes índices de sequía, que presentan una serie de ventajas e inconvenientes bajo distintas condiciones climáticas (Mishra \&y Singh, 2011; Marcos, 2001). Entre ellos se pueden destacar el Palmer Drouhgt Severity Index (PDSI) (Palmer, 1965), Standardized Precipitation Index (SPI) (Mckee et al., 1993) o Índice Estandarizado de Sequía Pluviométrica (IESP) (Pita,2000). Sin duda, el SPI es el más utilizado en el ámbito científico, debido a que únicamente requiere datos de precipitación, la simplicidad de su cálculo respecto a otros índices, y la comparabilidad entre unas regiones y otras al ser un índice adimensional (Santos et al., 2010; He et al., 2015). En los últimos años se ha introducido la variable de la temperatura en el análisis de la sequía meteorológica para analizar el impacto del aumento de las temperaturas en este fenómeno. Para ello se propuso el índice Standardized Precipitation Evapotranspiration Index-SPEI (Vicente et al., 2010).

En la península ibérica, se han llevado a cabo numerosos trabajos sobre tendencias de las precipitaciones y la evolución de los periodos de sequía. Durante la segunda mitad del S.XX se muestra una clara tendencia negativa de las precipitaciones en la vertiente atlántica (SerranoPérez et al., 1999; González et al., 2011; Ríos et al., 2013; Serrano-Notivoli, 2017). Sin embargo en la vertiente mediterránea si se observan tendencias ligeramente ascendentes en algunas áreas (De Luis et al., 2009; Bartolomeu et al., 2016; Serrano-Notivoli, 2017). En estudios a escala regional, concretamente en las demarcaciones hidrográficas del Segura y Júcar, también se vislumbran tendencias positivas en áreas de montaña del interior, aunque estas no son estadísticamente significativas (Miró et al., 2018).

Con respecto, al número de días de lluvia se observa un incremento durante el S.XX en la península ibérica (Rodrigo \& Trigo; 2007; Gallego et al., 2011). En Serrano-Notivoli (2017), se 
constata una tendencia positiva del número de días de lluvia (NWD), siendo esta especialmente intensa y significativa en toda la costa mediterránea española. Además, en España se ha observado un incremento casi generalizado en el índice de concentración de precipitación diaria (Cl), lo cual puede llevar consigo un aumento en muchos riesgos ambientales como la erosión o las inundaciones (Martín Vide, 2004; Serrano-Notivoli et al., 2018a). Por otra parte, en lo referido a la evolución de los periodos de sequía los estudios muestran una intensificación de los mismos en el ámbito de las regiones mediterráneas (Sousa et al., 2011; González et al., 2018; Domínguez et al., 2019). En cambio, se observa una reducción en la duración media de las rachas secas en España, siendo espacialmente marcado este descenso en la vertiente mediterránea (Serrano Notivoli et al., 2018b).

Por otro lado, en el conjunto de la sociedad y en el ámbito científico, existe una elevada preocupación por los impactos futuros del cambio climático sobre los recursos hídricos. Recientes investigaciones evidencian una clara intensificación de los fenómenos hidrológicos extremos en Europa (Lehner et al., 2006; Rajczak et al., 2013). Según las estimaciones de las proyecciones regionalizadas de cambio climático, las regiones mediterráneas serán uno de los ámbitos territoriales más afectados por la intensificación de los periodos de indigencia pluviométrica (Stagge et al., 2015; Spinoni et al., 2018). En este sentido, los avances en la previsión estacional de las sequías y en alerta temprana jugarán un papel fundamental en la minimización de los impactos de este fenómeno tanto a escala global como regional (García Galiano et al., 2007; Camarillo, 2012; Turco et al., 2017; Vicente et al., 2017; Pontes et al., 2019). Ante estos escenarios, los estudios de regionalización resultan de vital importancia con el objetivo de monitorizar diferentes regímenes de sequía y su distribución espacial con el fin de analizar más a fondo el comportamiento estacional de las sequías y poder articular de esta manera medidas encaminadas a la reducción de los impactos territoriales, económicos, sociales, políticos y medioambientales (Alvarez, 2004; Poquet et al., 2008; Santos et al., 2010; Portela et al., 2015).

El principal objetivo de este trabajo es realizar una caracterización de las sequías en los ámbitos territoriales de la Demarcación Hidrográfica del Segura (DHS) y la Mancomunidad de los Canales del Taibilla (MCT), localizados en el sureste de la península ibérica. La caracterización de sequías está basada en la aplicación del índice SPI a la mayor cantidad de series de precipitación posible. En este artículo se presentan los resultados obtenidos más relevantes, así como el marco conceptual y metodológico implementado que incluye los siguientes aspectos: (i) tratamiento de la series de precipitación mensual (detección de inhomogeneidades y relleno de 
huecos); (ii) cómputo del SPI para una escala temporal de 12 meses (periodo más idóneo para la evaluación de las sequías hidrológicas); (iii) propuesta de regionalización de sequía mediante la aplicación de un análisis de componentes principales (ACP); (iv) análisis de la frecuencia anual de ocurrencia de sequía a través de la utilización del método Kernel Ocurrence Rate Estimation (KORE) para cada región homogénea; (v) caracterización y evolución temporal del área afectada por sequías para cada región homogénea.

\section{Metodología}

\section{1 Área de estudio}

El ámbito territorial estudiado se encuentra ubicado en el sureste de la península ibérica (figura 1). Ocupa una superficie de $19697 \mathrm{~km}^{2}$ y cuenta con una población de 2633315 habitantes a 1 de enero de 2018 (Instituto Nacional de Estadística-INE). Se ha seleccionado el marco espacial de la DHS y MCT debido a que son los organismos responsables de la gestión y la planificación de los recursos hídricos, y del abastecimiento de agua potable, respectivamente, de gran parte del sureste de España. El espacio geográfico de la DHS es uno de los territorios más áridos de Europa, siendo la única de un total de 215, cuya precipitación anual es inferior a $450 \mathrm{l} / \mathrm{m}^{2}$ (MAPAMA). Por lo tanto, este territorio posee una situación de escasez pluviométrica estructural, lo que deriva en un déficit hídrico igualmente estructural. La MCT abastece a 80 municipios que engloban un total de 2477058 habitantes. Los recursos de la MCT proceden fundamentalmente de tres fuentes de abastecimiento: embalse del Taibilla, Trasvase Tajo-Segura y desalinización (4 plantas desalinizadoras ubicadas en los litorales de las provincias de Murcia y Alicante).

Una de las principales características del relieve del área de estudio es la elevada variabilidad orográfica que presenta (figura 1). De este modo, se alternan sistemas montañosos que superan los 2000 metros de altitud (zona noroccidental) con valles, altiplanicies, depresiones y llanuras costeras. Estos acusados contrastes en el relieve derivan de la combinación de múltiples factores estructurales, tectónicos y climáticos. Esto es consecuencia de la ubicación del sureste de España en uno de los sistemas morfoestructurales más complejos de la península ibérica: las Cordilleras Béticas (Conesa, 2006). La disposición del relieve juega un papel primordial en la elevada variabilidad climática existente en este territorio. La ubicación geográfica del sureste de España provoca que se vea afectado por situaciones atmosféricas muy diversas, asociadas fundamentalmente a la circulación zonal y meridiana de flujos de aire tropical y aire polar, siendo predominantes las masas de aire cálido tropical modificadas en su recorrido por el mar Mediterráneo (Gil, 2007). A su vez, durante gran parte del año, se encuentra sometido a la 
acción de abrigo aerológico que ejerce el Anticiclón de las Azores, responsable de largos e intensos periodos de sequía (Font, 1988).

\section{Figura 1. Área de estudio}

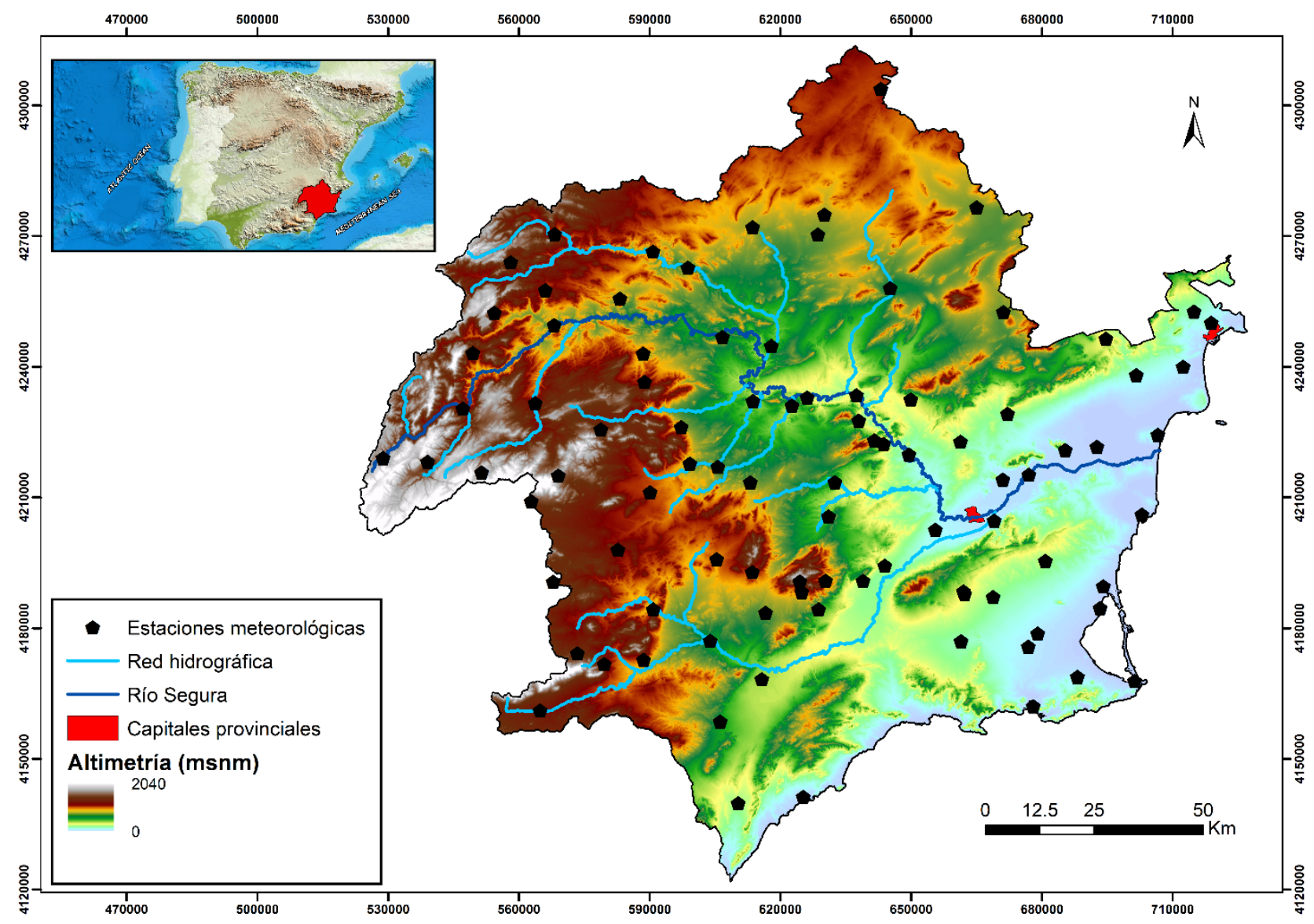

Fuente: elaboración propia

Con respecto a la precipitación, hay que destacar que cuenta con una elevada variabilidad interanual, la cual se constituye como una de las principales características del clima mediterráneo y los climas semiáridos. De este modo se suceden largos periodos secos, con episodios de lluvias torrenciales, que provocan graves inundaciones (Olcina, 2001). Existe una clara relación entre la distribución espacial de la precipitación media anual y el relieve, de modo que los máximos pluviométricos se registran en los relieves noroccidentales donde superan puntualmente los $1000 \mathrm{l} / \mathrm{m}^{2} /$ año (Figura 2). En este sector los frentes atlánticos se encuentran con relieves superiores a 1500 metros de altitud, por lo que las masas de aire se ven obligadas a ascender (disparo orográfico) originando importantes precipitaciones a barlovento de dicho flujo. En el resto del área de estudio, localizada a sotavento de dicho flujo húmedo atlántico, los frentes llegan bastante degradados debido a la acción de barrera orográfica (efecto Foehn) de relieves como la sierra de Segura (Capel, 1983). 
De esta forma, se produce un marcado descenso gradual de las precipitaciones desde el extremo noroccidental donde se superan los $1000 \mathrm{l} / \mathrm{m}^{2}$ hasta el extremo suroccidental donde el acumulado medio anual es inferior a $200 \mathrm{l} / \mathrm{m}^{2}$ (Figura 2). El gradiente altitudinal entre estos dos puntos es 58,1 mm/100 m. El litoral suroccidental de la Región de Murcia junto con el litoral de Almería se constituye como la zona más árida del continente europeo. Los relieves situados en la parte central del área de estudio se configuran como auténticas islas de humedad. Las precipitaciones se registran fundamentalmente durante los equinoccios, concentrándose estas durante muy pocos días en forma de lluvias torrenciales (Morales et al., 1999). Una clara muestra de la elevada torrencialidad y variabilidad de las precipitaciones se encuentra en los acumulados máximos en 24 horas. Los récords de precipitación máxima diaria se localizan en observatorios situados en el litoral oriental, debido a su disposición favorable a barlovento de los vientos húmedos mediterráneos. El 4 de noviembre de 1987 se registró una precipitación de 330 l/m² en San Javier y el 30 de septiembre de 1997, 270,2 I/m² en Alicante (Garrido et al., 2014; Benhamrouche \& Martín Vide, 2011).

El arco montañoso noroccidental supone una excepción a este patrón, estableciéndose como la única zona donde se produce el máximo estacional durante el inverno algo característico del clima mediterráneo (Conesa \& Alonso, 2006). En el resto del territorio, el otoño es la estación más húmeda. Por otra parte, la sequía estival es muy marcada en la zona meridional del área de estudio. En la zona septentrional (zona de mayor altitud) el estío no es tan seco debido al desarrollo de fenómenos termoconvectivos que dan lugar a chubascos tormentosos. Por último, en Miró et al. (2018) se realiza un análisis de los cambios de los tipos de precipitación (frentes atlánticos, génesis mediterránea y precipitación convectiva) en los últimos 60 años en el territorio de la DHS, concluyendo que existe una clara tendencia descendente de la precipitación de origen atlántico lo cual afecta en mayor medida al sector noroccidental. En cambio, se observan tendencias ligeramente positivas en algunas zonas montañosas de interior de las precipitaciones de origen convectivo y mediterráneo. Estas variaciones pueden influir en los patrones espaciales de las sequías 
Figura 2. Distribución espacial de la precipitación media anual

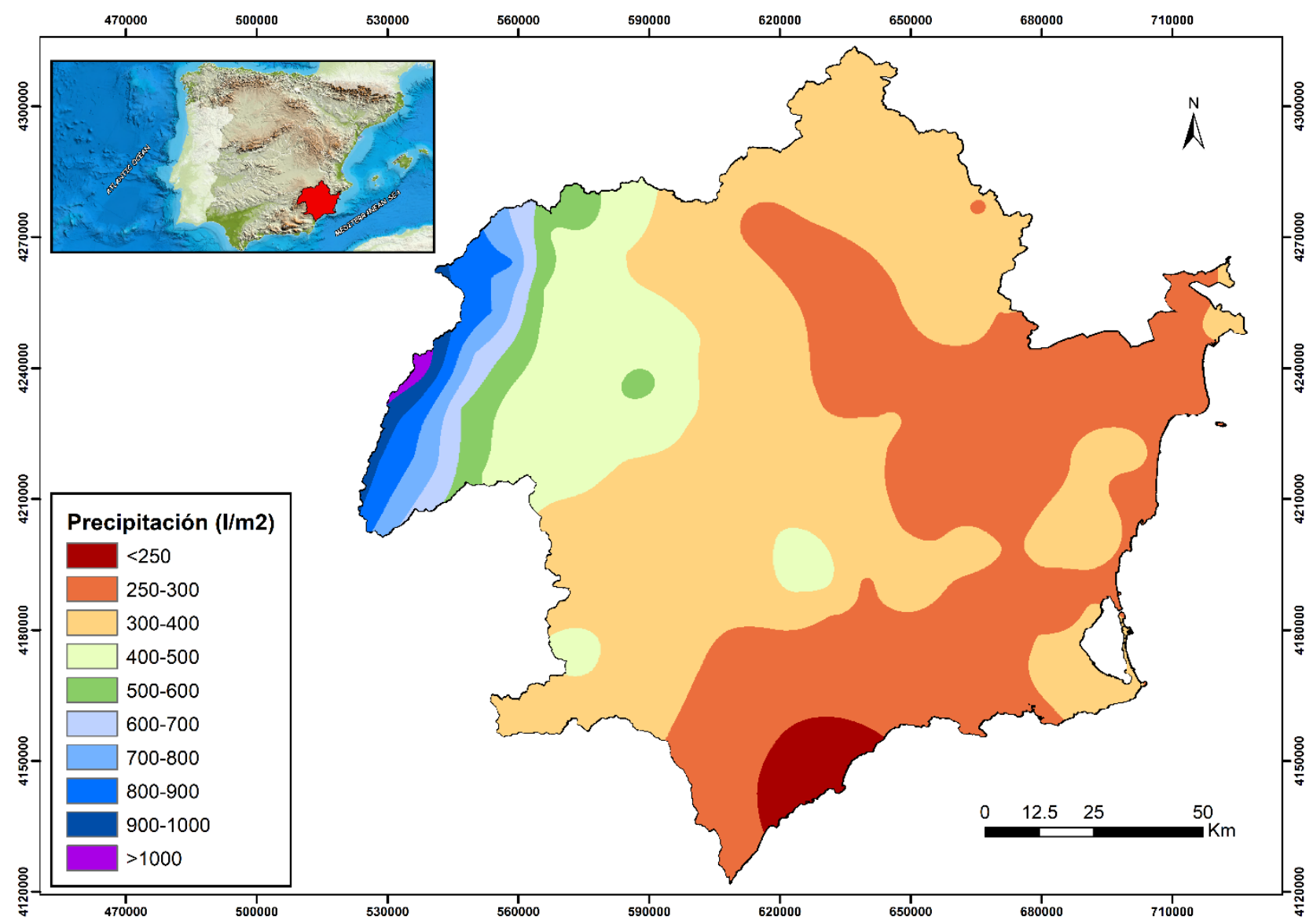

Fuente: elaboración propia a partir de los datos de la Agencia Estatal de Meteorología (AEMET)- periodo de referencia 1981-2010

\subsection{Base de datos de precipitación. Proceso de control de calidad y relleno de lagunas}

La base de datos utilizada en este estudio cuenta con un total de 92 series de precipitación mensual procedentes de los registros de la Agencia Estatal de Meteorología (AEMET). Su distribución espacial se muestra en la figura 1. El periodo temporal seleccionado es 1968-2017, con el objetivo de poder incluir el mayor número de estaciones posibles. Las principales características de las series de precipitación se muestran en la tabla 1. Para el proceso de control de calidad y relleno de datos faltantes de las series se ha utilizado fundamentalmente el paquete de R "reddPrec" (https://CRAN.R-project.org/package=reddPrec) implementado recientemente por Serrano-Notivoli et al. (2017). Es un paquete que posibilita la reconstrucción de la precipitación a escala diaria. No obstante, para la detección de inhomogeneidades se ha realizado un primer control de calidad con el paquete de R "CLIMATOL" (Guijarro, 2018) , con el objetivo de eliminar valores atípicos. Consecutivamente, la aplicación de las funciones del 
paquete "reddPrec" de forma secuencial permite realizar en primer lugar un control de calidad de los datos facilitando la eliminación de datos sospechosos. Posteriormente se realiza el relleno de lagunas sobre la base de datos ejecutada en el control de calidad. De esta forma, se obtienen las 92 series de precipitación homogéneas. El porcentaje de datos faltantes de cada serie se muestra en la tabla 1. Se han utilizado algunas series con un \% de lagunas entre un 20$25 \%$ con el objetivo de cubrir zonas con ausencia de registros, sobre todo en la cabecera del Segura. Por último, el mapa de distribución espacial de la precipitación anual (figura 2), ha sido realizado a partir de los registros de un total de 137 series de precipitación de la base de datos de AEMET para el periodo de referencia 1981-2010. La elaboración del mapa se ha llevado a cabo con el método Kriging Ordinario, implementada en el software ArcGIS.

Tabla 1. Características de las 92 series de precipitación utilizadas

en el proceso de regionalización

\begin{tabular}{|c|c|c|c|c|c|c|c|}
\hline ID & Nombre & $\begin{array}{l}\text { UTMX- } \\
30 N\end{array}$ & $\begin{array}{l}\text { UTMY- } \\
30 N\end{array}$ & $\begin{array}{l}\text { Altitud } \\
\text { (msnm) }\end{array}$ & $\begin{array}{c}\text { Región } \\
\text { homogénea }\end{array}$ & $\begin{array}{c}\% \\
\text { huecos } \\
\end{array}$ & $P\left(I / m^{2}\right)$ \\
\hline 7119A & Caravaca & 599265 & 4217785 & 623 & RG3 & 1,4 & 384,1 \\
\hline 7129 & Emb. Alfonso XIII & 622719 & 4231114 & 312 & RG3 & 1,0 & 302,4 \\
\hline 7127 & Bullas & 613162 & 4213496 & 604 & RG1 & 2,5 & 392,7 \\
\hline 7168 & Emb. de la Cierva & 632540 & 4213541 & 395 & RG3 & 0,7 & 340,1 \\
\hline $7123 E$ & Inazares & 569086 & 4215068 & 1300 & RG3 & 2,9 & 470,8 \\
\hline 7113 & Campo de S. Juan & 578851 & 4225636 & 1150 & RG3 & 1,4 & 491,4 \\
\hline $7114 C$ & Moratalla & 597290 & 4225636 & 710 & RG3 & 3,3 & 419,4 \\
\hline 7083 & Emb. del Cenajo & 606750 & 4246858 & 360 & RG3 & 0,4 & 392,5 \\
\hline $7138 \mathrm{~B}$ & Jumilla & 645247 & 4258045 & 485 & RG3 & 0,4 & 297 \\
\hline 7215 & Totana Carrasca & 624526 & 4190755 & 1200 & RG1 & 3,1 & 467,1 \\
\hline 7216 & Totana Morti & 628806 & 4184347 & 480 & RG1 & 1,7 & 316,8 \\
\hline 7219 & Alhama-Espuña & 630390 & 4190908 & 760 & RG1 & 0.1 & 423,7 \\
\hline 7151 & Abarán $S^{a}$ del Oro & 638029 & 4227661 & 400 & RG3 & 6,7 & 305,6 \\
\hline $7250 \mathrm{C}$ & Abanilla & 672171 & 4229217 & 174 & RG4 & 3,1 & 287 \\
\hline 7237 & Fortuna & 661370 & 4222828 & 192 & RG3 & 1,2 & 267,8 \\
\hline $7023 x$ & Fuente Álamo & 661516 & 4177040 & 140 & RG1 & 0,6 & 277,7 \\
\hline 7031 & San Javier & 693425 & 4184666 & 4 & RG1 & 0,2 & 313 \\
\hline $7211 \mathrm{~B}$ & Puerto Lumbreras & 606219 & 4158572 & 445 & RG1 & 5,0 & 274,5 \\
\hline 7226 & Librilla & 643994 & 4194373 & 180 & RG1 & 1,2 & 300,9 \\
\hline 7156 & Ricote & 641612 & 4223128 & 480 & RG3 & 0,6 & 335 \\
\hline 7275 & Yecla & 665085 & 4276585 & 605 & RG3 & 3,1 & 296,3 \\
\hline $7145 B$ & Cieza & 637517 & 4233449 & 188 & RG3 & 0,5 & 258,9 \\
\hline 7198 & Emb.Valdeinfierno & 590892 & 4184364 & 690 & RG1 & 0,2 & 355,1 \\
\hline 7206 & Zarzadilla Totana & 613570 & 4192908 & 861 & RG1 & 2,5 & 350,5 \\
\hline $7201 \mathrm{E}$ & Avilés & 605419 & 4195882 & 770 & RG1 & 7,7 & 296,2 \\
\hline 7205 & Emb. de Puentes & 603946 & 4177121 & 450 & RG1 & 0,5 & 302,5 \\
\hline 7209 & Lorca & 615799 & 4168318 & 312 & RG1 & 4,7 & 252,2 \\
\hline 7026 & Pozo Estrecho & 676924 & 4175783 & 50 & RG1 & 0,7 & 296,1 \\
\hline 7016 & Cartagena El Algar & 688204 & 4168878 & 40 & RG1 & 3,3 & 370,3 \\
\hline 7019 & Salinas Cabo Palos & 701322 & 4167913 & 1 & RG1 & 10,6 & 302,9 \\
\hline 7013 & Cartagena Puerto & 678051 & 4162116 & 10 & RG1 & 4,3 & 272,5 \\
\hline 7228 & Murcia/Alcantarilla & 655618 & 4202611 & 75 & RG1 & 0 & 289,5 \\
\hline 7120 & Cehegín & 605682 & 4217064 & 563 & RG3 & 13.4 & 392,1 \\
\hline 7002 & Águilas & 625273 & 4141287 & 20 & RG1 & 0.4 & 204,1 \\
\hline $7161 \mathrm{~A}$ & Archena & 649537 & 4119910 & 100 & RG3 & 0.2 & 266,8 \\
\hline 7159 & Blanca Casa Castillo & 649986 & 4232498 & 280 & RG3 & 6,1 & 282,9 \\
\hline 7170 & Pliego & 631107 & 4205656 & 381 & RG1 & 2,8 & 367,5 \\
\hline 7032 & S. Pedro del Pinatar & 694138 & 4189639 & 19 & RG1 & 5,7 & 293,4 \\
\hline
\end{tabular}


Tabla 1. Continuación

\begin{tabular}{|c|c|c|c|c|c|c|c|}
\hline ID & Nombre & $\begin{array}{l}\text { UTMX- } \\
30 N\end{array}$ & $\begin{array}{l}\text { UTMY- } \\
30 N\end{array}$ & $\begin{array}{l}\text { Altitud } \\
\text { (msnm) }\end{array}$ & $\begin{array}{c}\text { Región } \\
\text { homogénea }\end{array}$ & $\begin{array}{c}\% \\
\text { huecos }\end{array}$ & $P\left(I / m^{2}\right)$ \\
\hline 7232 & Santomera & 671099 & 4214137 & 36 & RG1 & 0,9 & 276,3 \\
\hline 7157A & Ulea & 643721 & 4222302 & 110 & RG3 & 6,7 & 302,9 \\
\hline 7231 & Beniaján & 669099 & 4204720 & 50 & RG1 & 3,2 & 282,1 \\
\hline $7221 \mathrm{~F}$ & Alhama El Praico & 639019 & 4190895 & 210 & RG1 & 3,7 & 293,2 \\
\hline $7118 \mathrm{~B}$ & Barranda & 590131 & 4211236 & 850 & RG1 & 1,3 & 355,5 \\
\hline 7121 & Calasparra & 613754 & 4232124 & 340 & RG3 & 1,8 & 354,5 \\
\hline 7195 & Los Royos & 582760 & 4198035 & 985 & RG1 & 6,8 & 335,4 \\
\hline 7131 & Cieza Almadenes & 626193 & 4232925 & 200 & RG3 & 0,3 & 314,1 \\
\hline 7207 & Lorca Juncosa & 616635 & 4183549 & 580 & RG1 & 1,9 & 315,5 \\
\hline 7190 & Lorca Tirieza Alta & 588620 & 4172780 & 790 & RG1 & 5,2 & 346,2 \\
\hline 7080 & Benizar & 588854 & 4236529 & 899 & RG3 & 1,5 & 539,2 \\
\hline 5057 & Cañada de la Cruz & 562870 & 4209161 & 1271 & RG3 & 1,1 & 433 \\
\hline 7029 & Murcia Sucina & 680849 & 4195504 & 203 & RG1 & 0,7 & 308,6 \\
\hline 7020 & Murcia Corvera & 662073 & 4188481 & 280 & RG1 & 0,5 & 284,2 \\
\hline 7021 & Murcia S. Magin & 662304 & 4187931 & 260 & RG1 & 0 & 286,4 \\
\hline 7025 & Martínez del Puerto & 668874 & 4187200 & 160 & RG1 & 3,2 & 266,6 \\
\hline 7214 & Totana Alquerías & 624929 & 4188356 & 799 & RG1 & 3,6 & 387,5 \\
\hline $7026 x$ & Torre Pacheco & 679088 & 4178790 & 50 & RG1 & 8,6 & 299,7 \\
\hline 7104 & Albatana & 628633 & 4270364 & 580 & RG3 & 3 & 281,5 \\
\hline 7059 & Arguellite & 549472 & 4243127 & 980 & RG2 & 3,2 & 705,8 \\
\hline 7089 & Bogarra & 568250 & 4270364 & 880 & RG2 & 2,3 & 469,5 \\
\hline 7268 & Bonete & 643111 & 4303790 & 888 & RG3 & 14,4 & 376,7 \\
\hline $7067 Y$ & Cañadas de Nerpio & 551470 & 4215779 & 1485 & RG2 & 19,8 & 501,8 \\
\hline 7077 & Elche de la Sierra & 583237 & 4255674 & 630 & RG3 & 1,9 & 412,9 \\
\hline $7109 Y$ & Emb. de Camarillas & 617971 & 4244764 & 397 & RG3 & 11,9 & 327,6 \\
\hline 7066 & Emb. Fuensanta & 568112 & 4249583 & 680 & RG3 & 2,8 & 441,4 \\
\hline 7071 & Presa del Taibilla & 563790 & 4231669 & 870 & RG2 & 1,1 & 420,6 \\
\hline 7094 & Emb. del Talave & 598811 & 4262757 & 550 & RG3 & 16,7 & 375,5 \\
\hline 7092 & Liétor & 590800 & 4266454 & 641 & RG3 & 1,7 & 386,4 \\
\hline 7065 & Molinicos & 566106 & 4257581 & 823 & RG2 & 1,8 & 486,2 \\
\hline 7102 & Ontur & 630156 & 4274922 & 670 & RG3 & 1,8 & 323,4 \\
\hline 7081 & Socovos & 588540 & 4243061 & 750 & RG3 & 3,3 & 468,3 \\
\hline 7103 & Tobarra & 613674 & 4272049 & 625 & RG3 & 0,4 & 284,1 \\
\hline 7086 & Cañada Provencio & 558134 & 4263992 & 1125 & RG2 & 8,8 & 819,1 \\
\hline 7064 & Yeste Hoya Parilla & 554386 & 4252376 & 1018 & RG2 & 22,4 & 797,1 \\
\hline 7045 & Pontones & 528848 & 4219079 & 1333 & RG2 & 7,2 & 871,7 \\
\hline 7054 & Salto de Miller & 547191 & 4230414 & 725 & RG2 & 0,9 & 588,2 \\
\hline 7056 & Stgo. de la Espada & 539104 & 4218228 & 1332 & RG2 & 4,6 & 753,1 \\
\hline 7187A & Chirivel & 564906 & 4161125 & 1046 & RG1 & 10,5 & 314,1 \\
\hline 7194 & María & 573447 & 4174234 & 1202 & RG1 & 4,0 & 452,6 \\
\hline 6372 & Pulpi Canalejas & 610366 & 4139843 & 180 & RG1 & 9,6 & 254,9 \\
\hline 7185 & Vélez Blanco & 579617 & 4171794 & 1080 & RG1 & 7,3 & 383,9 \\
\hline $5060 x$ & Topares & 567903 & 4190655 & 1182 & RG1 & 2,3 & 322 \\
\hline $7038 C$ & Torrevieja & 703003 & 4206087 & 9 & RG1 & 9,5 & 281,6 \\
\hline 7247 & Pinoso & 671237 & 4252574 & 575 & RG3 & 0,6 & 286,1 \\
\hline 7256 & Callosa de Segura & 685406 & 4220935 & 25 & RG4 & 2,7 & 296,3 \\
\hline 7261 & Almoradí & 692698 & 4221660 & 7 & RG1 & 18,7 & 311,2 \\
\hline 8017 & Aspe & 694757 & 4246446 & 241 & RG4 & 8,0 & 296,8 \\
\hline 8018A & Elche & 701719 & 4238104 & 95 & RG4 & 0,7 & 271,9 \\
\hline 8019 & Alicante/El Altet & 712468 & 4239983 & 43 & RG4 & 0,4 & 276,4 \\
\hline 8023 & S.Vicente Raspeig & 714974 & 4252698 & 115 & RG4 & 1,5 & 286,4 \\
\hline 8025 & Alicante & 718904 & 4250120 & 81 & RG4 & 0,0 & 311,1 \\
\hline 7244 & Orihuela & 677074 & 4215260 & 26 & RG1 & 1,6 & 284,4 \\
\hline 7263 & La Marina de Elche & 706665 & 4224378 & 15 & RG1 & 6,3 & 289,9 \\
\hline
\end{tabular}

Fuente: elaboración propia a partir de la base de datos de la Agencia Estatal de Meteorología (AEMET) 


\subsection{Standardized Precipitation Index (SPI)}

La elección del índice de sequía depende de múltiples factores como las características del régimen hidrológico o las variables disponibles. En este estudio se ha seleccionado el SPI al ser el índice más utilizado en el ámbito científico (Agnew, 2000; Portela et al., 2015; Espinosa et al., 2019). El SPI se calcula a partir de la conversión de datos de precipitación (se recomiendan más de 30 años de observación) a probabilidades basadas en registros de precipitación acumulada. Dichas probabilidades componen series normalizadas con un promedio de 0 y una desviación estándar de 1 (Peña et al., 2016). La principal utilidad de este índice es que facilita el análisis de los impactos de las sequías en distintas escalas temporales (1, 3, 6, 9, 12, 24 y 48 meses). Por lo tanto, permite la identificación de los distintos tipos de sequía (meteorológica, agrícola, hidrológica y socioeconómica) definidos por Wilhite y Glantz (1985), ya que los diferentes sistemas naturales y sectores económicos pueden responder a las condiciones de sequía en escalas temporales desiguales (Vicente et al., 2012). Un periodo inferior o igual a 3 meses permite la monitorización de las sequías meteorológicas, por encima de 6 meses se pueden evaluar las sequías agrícolas, y por último, el periodo de 12 meses es el ideal para evaluar las sequías hidrológicas. Este periodo temporal de 12 meses, presenta cierta variabilidad, ya que no todos los ríos responden a la misma escala temporal de sequía.

En este trabajo se utiliza el SPI12, que es considerado como un índice de sequía hidrológica (Szalai \& Szinell, 2000). Se ha seleccionado está escala temporal (12 meses) debido a las repercusiones territoriales que tiene este tipo de sequía en el sureste de la península ibérica, área que cuenta con un importante déficit hídrico estructural. Con el objetivo de agrupar sequías mutuamente dependientes y eliminar sequías cortas con poca repercusión hidrológica se ha aplicado un filtro de paso bajo de 5 meses a los valores originales del SPI (López-Moreno et al., 2009). Además, puede ser de gran utilidad para reducir el efecto sobre la intensidad de sequía de las precipitaciones intensas de corta duración típicas del clima del sureste peninsular. De esta forma, se obtienen mejores resultados que con otros métodos (Fleig et al., 2006). Por último, para clasificar las diferentes categorías de sequías se emplea la clasificación modificada por Agnew (2000), debido a que sus valores se ajustan a una clasificación más racional (Portela et al., 2015; Santos et al., 2010) que la propuesta original de Mckee et al. (1993). Con esta clasificación los valores de intensidad por debajo de -0,84 representan sequía moderada, por debajo de -1,28 sequía severa y por debajo de -1,65 sequía extrema, que se configuran como las secuencias pluviométricas secas de mayor severidad. Por otra parte, entre $-0,84$ y 0,84 se consideran valores normales y por encima de 0,84 se localiza la categoría de húmedo. 


\subsection{Análisis de componentes principales (ACP) y otros métodos de clúster}

Las sequías constituyen riesgos naturales regionales, lo cual implica que sea muy importante la identificación de áreas homogéneas donde el comportamiento de los patrones espaciotemporales de las sequías (frecuencia de ocurrencia, severidad o duración) sean similares. En este trabajo, para la identificación de regiones homogéneas se ha aplicado la técnica de análisis de componentes principales (ACP en adelante) a las series temporales suavizadas del SPI12. Este método ha sido utilizado por numerosos autores en ámbitos climáticos muy diferentes con resultados satisfactorios (Vicente et al., 2004; Santos et al., 2010; Portela et al., 2015; Espinosa et al., 2019).

ACP es una técnica multivariante que analiza una base de datos cuyas observaciones son descritas por varias variables dependientes intercorrelacionadas (Abdi \& Williams, 2010). Esta técnica permite descomponer el conjunto de datos de una variable dada (en este caso SPI12) en representaciones univariadas de esa variable (Castell et al., 2006). De este modo, las variables originales correlacionadas se pueden agrupar en un número mucho más reducido de variables linealmente no correlacionadas, las cuales explican la mayor parte de la varianza total (Vicente et al., 2004). En definitiva, se pretende reducir la dimensionalidad de los datos (Hair et al., 1998).

En el presente trabajo, el análisis de ACP ha consistido en el cálculo de la matriz de covarianza de los datos del SPI12 para los correspondientes valores propios ("eigenvalues") y vectores propios ("eigenvectors") (Richman, 1986). Los componentes principales (CP en adelante) están dados por la proyección de los campos del SP12 en las propias funciones ortonormales (Raziei et al., 2015). Por otra parte, cabe mencionar que la identificación de los patrones espaciales del SPI12 está basada en la carga de los CP, los cuales representan la correlación entre cada una de las series temporales de SPI12 (obtenidas a partir de las series de precipitación) y la correspondiente serie de componente principal. Estas series de componentes principales se configuran como series "regionalizadas" de SPI12. Por último, para llevar a cabo una mejor interpretación de los resultados obtenidos, los principales componentes han sido rotados, utilizando la técnica Varimax, que es ampliamente utilizada en el ámbito científico para realizar estas rotaciones (Richman, 1986). De este modo se consigue localizar con mayor precisión la variabilidad espacial de los patrones de sequía. Los patrones obtenidos a partir de este método se denominan componentes principales rotados (CPR en adelante).

Para finalizar, es muy importante resaltar que la identificación de los grupos homogéneos es muy dependiente de la técnica de clúster utilizada. Por tanto, en este trabajo, aparte del ACP, se han 
implementado otras técnicas de clúster, para corroborar y dar estabilidad a los grupos obtenidos con el análisis de ACP. Las técnicas de clúster implementas son el método jerárquico Ward's con la distancia Euclidiana, y el método no jerárquico K-Means, debido a que son dos de las técnicas que proporcionan mejores resultados (Hair et al., 1998). Ambas técnicas han sido implementadas en R. En el caso del método Ward's con la distancia Euclidiana se ha estimado el número de regiones óptimas, resultando el mismo número de regiones que en el $\mathrm{ACP}$. Por su parte, en el método de K-Means se ha establecido el número de regiones obtenido en las otras técnicas utilizadas. En definitiva, aunque el análisis por clúster se rige por un análisis discriminante para verificar la significancia estadística de los grupos obtenidos, no se garantiza que los grupos sean realmente significativos, de ahí la utilidad de utilizar más de una técnica de clúster (Dillan \& Goldstein, 1986).

\subsection{Frecuencia de ocurrencia de sequías}

La frecuencia es un parámetro de sequía que puede ser descrita como el número de eventos que se produce durante un periodo de tiempo determinado. Su caracterización requiere del análisis de series de observación suficientemente largas, y suele expresarse por medio de su probabilidad de ocurrencia o período de retorno (Pita, 1989). En este estudio para analizar los cambios en la frecuencia de ocurrencia de sequías se ha utilizado el método Kernel Ocurrence Rate Estimator (KORE) de acuerdo con la metodología implementada por Silva et al. (2012) y Portela et al. (2015), incluyendo la generación de pseudodatos antes y después del periodo de observación, a través del método de reflexión para una amplitud de tres veces el ancho de banda (Portela et al., 2015; Espinosa et al., 2019). Para la selección de dicho ancho de banda (h), se ha aplicado la regla general de Silverman (Silverman, 1986).

La técnica Kernel es un método no paramétrico desarrollado por Diggle (1985) para el suavizado de procesos puntuales de datos. El principal objetivo de la aplicación del KORE es analizar cómo cambia la frecuencia anual de los periodos bajo condiciones de sequía, $\lambda(t)$, a través del tiempo. Con esta finalidad, el análisis KORE ha sido aplicado a los valores de las nuevas series regionalizas (CPR) del SPI12 (obtenidas en el proceso de ACP) que representen valores de sequía moderada o inferiores, es decir los registros inferiores a una intensidad de sequía de 0,84. Por último, con el objetivo de cuantificar la incertidumbre de los resultados obtenidos en este proceso, se han construido bandas de confianza alrededor de $\lambda(t)$, mediante las simulaciones Bootstrap (Mudelsee, 2011). Este método fue implementado por primera vez en el análisis de extremos climáticos por Mudelsee et al. (2003). 


\subsection{Evolución temporal del porcentaje de superficie afectadas por sequías}

Para cada una de las regiones obtenidas se ha realizado un análisis de la evolución temporal del porcentaje de área afectada por sequías con diferentes grados de severidad de acuerdo con las categorías del SPI anteriormente descritas (moderada, severa y extrema). El área de cada región atribuida a las diferentes categorías de sequía ha sido asignada mediante la aplicación de áreas acumulativas a partir de los polígonos de Thiessen (Thiessen, 1911). El objetivo de este apartado es poder estudiar posibles patrones espaciales de sequías, y ver que regiones han sido más afectadas por los diferentes ciclos secos. Además, la identificación del área afectada por sequías con diferentes grados de severidad puede ser de gran ayuda a la hora de evaluar los daños ocasionados por dicho evento natural.

\section{Resultados}

\subsection{Regiones homogéneas a partir de los componentes principales rotados y Kernel Ocurrence Rate Estimation (KORE)}

En la figura 3 se muestran los diez primeros valores propios resultantes del análisis ACP aplicado a las series suavizadas del SPI12. El "scree plot" ha sido el primer criterio utilizado para seleccionar el número de CP a retener (Hair et al., 1998). Se observa una línea que deja de descender y se estabiliza aproximadamente en la cuarta $C P$, lo que significa que entre tres o cuatro componentes principales deben ser retenidos. El mapeo de los valores de las correlaciones entre las series SPI12 y las series CPR ("factor loadings"), sugirió la selección de los cuatro primeros componentes, ya que cubren completamente el área de estudio, y no se superponen (Figura 4). La selección de las cuatro CP fue corroborada con la aplicación de otras técnicas clúster (Ward's Eucledian y K-Means), y la aplicación del análisis KORE a cada una de las series suavizadas SPI12 para compararlas con el KORE de las series CPR.

Mediante el uso de los valores propios puede ser extraída información del porcentaje de varianza explicada por cada uno de los CP, tanto a nivel individual como la varianza acumulada. En la Tabla 2 se muestra la varianza explicada por las diez primeras CP (no rotados y rotados). El porcentaje de varianza acumulada total explicada por las cuatro primeras componentes es de un 82,2\%, distribuyéndose de la siguientes forma: 68 \% (CP1), 8,1\% (CP2), 3,3\% (CP3) y 2,8\% (CP4). A partir de la cuarta PC, la contribución de la varianza de cada nueva dimensión es insignificante (todas las variables inferiores a un $2 \%$ ). De esta forma, solo las cuatro primeras CP han sido retenidas para la ejecución de la rotación por Varimax, con el objetivo de identificar 
cuatro regiones homogéneas de SPI12. Una vez realizada la rotación se observa como desciende considerablemente la varianza explicada por el CP1, aumentando este valor en el resto de las componentes (CP2, CP3 y CP4).

Figura 3. Diez primeros valores propios resultantes

del análisis de ACP aplicado a las series de SPI12

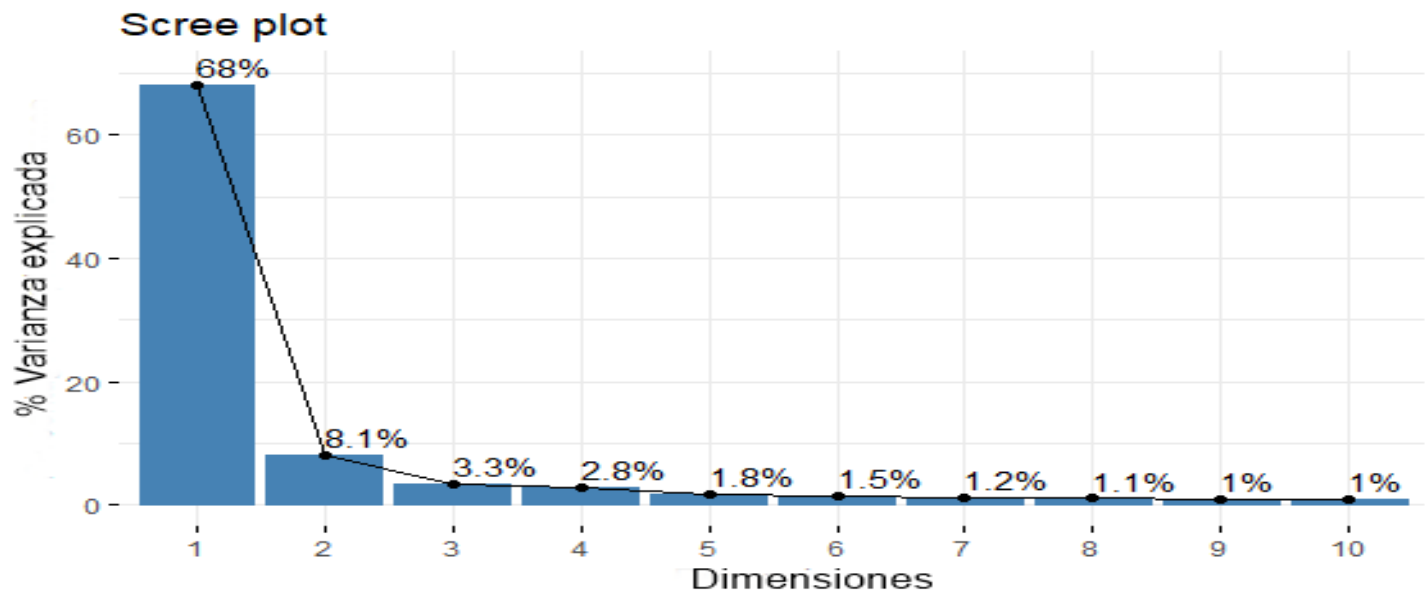

Fuente: elaboración propia a partir de análisis ACP en R

Tabla 2. Porcentaje de varianza explicada parcial y acumulada de los componentes de ACP (CP1 a CP10) rotados y no rotados

\begin{tabular}{|c|c|c|c|c|}
\hline \multirow{2}{*}{ ACP } & \multicolumn{4}{|c|}{ SPI12 } \\
\cline { 2 - 5 } & Varianza parcial explicada (\%) & \multicolumn{3}{|c|}{ Varianza acumulada explicada (\%) } \\
\cline { 2 - 5 } & No rotado & Rotado & No rotado & Rotado \\
\hline CP1 & 68.0 & 31.2 & 68.0 & 31.2 \\
CP2 & 8.1 & 14.7 & 76.1 & 45.9 \\
CP3 & 3.3 & 23.1 & 79.4 & 69.0 \\
CP4 & 2.8 & 13.2 & 82.2 & 82.2 \\
CP5 & 1.8 & & 84.0 & \\
CP6 & 1.5 & & 85.5 & \\
CP7 & 1.2 & & 86.7 & \\
CP8 & 1.1 & & 87.8 & \\
CP9 & 1.0 & & 88.8 & \\
CP10 & 1.0 & & 89.8 & \\
\hline
\end{tabular}

Fuente: elaboración propia a partir de análisis ACP en $\mathrm{R}$

Los cuatro primeros CPR del SPI12, son representados espacialmente en el mapa de cargas factoriales (representan la correlación existente entre cada CPR y cada una de las 92 series suavizadas de SPI12), los cuáles son representados en la figura 4. El método de interpolación utilizado ha sido el Inverse Distance Weighting (IDW) con un exponente de 2. De esta forma, el 
proceso de rotación ortogonal (Varimax) empleado para la identificación de patrones espaciales permite una delimitación bastante precisa de las regiones con diferente variabilidad temporal de sequías. A partir de los resultados de la regionalización del ACP, se han delimitado las regiones donde los valores del coeficiente de correlación entre cada CPR y las series SPI12 son iguales o superiores a 0,6 (las áreas con correlación igual o superior a 0,6 han sido delimitadas en el mapa mediante una línea discontinua) Se ha seleccionado este coeficiente relativamente bajo debido a la elevada variabilidad espacial de las precipitaciones en el área de estudio.

La delimitación de regiones homogéneas resultante es bastante razonable desde un punto de vista climático, hidrológico y orográfico. No obstante, cabe mencionar que la elevada variabilidad climática y orográfica existente en el área de estudio (Figuras 1 y 2) provoca que la regionalización de sequías sea bastante compleja (Figura 4). La CPR1 (31,2\% de la varianza total) explica un área bastante homogénea (correlación superior a 0,7) localizada en la mitad meridional del área de estudio. La CPR2 (14,7\% de la varianza total) revela un área reducida en el extremo noroccidental que muestran correlaciones muy elevadas que en algunos puntos superan el 0,9. La CPR3 (23,1\% de la varianza total) muestra un área localizada en gran parte de la mitad septentrional, aunque con correlaciones inferiores a otras regiones (entre 0,6 y 0,7). Cabe mencionar que en esta CPR hay bastantes estaciones pluviométricas que presentan una correlación entre 0,55 y 0,6. Por último, la CPR4 (13,2\% de la varianza total) explica un área pequeña ubicada en el extremo oriental, presentando correlaciones superiores a 0,7 en algunos casos.

Además para cada una de las series CPR se ha aplicado el análisis KORE anteriormente detallado. Los diagramas resultantes se exponen entre las figuras 5 y 8 . Han sido incluidas todas las categorías de sequía, representadas por valores del SPI12 iguales o inferiores a -0,84. En los diagramas del KORE se representan: ratio de ocurrencia dependiente del tiempo, $\lambda$ ( $t$ ) (línea roja) y banda de confianza (área gris sombreada) de ocurrencia de sequía. Además se muestra la evolución de cada uno de SPI regionalizados a partir de las CPR. Por último, se distingue con una línea discontinúa el umbral de sequía adoptado $(-0,84)$, y con una serie de marcas negras verticales los puntos en el periodo temporal analizado por debajo del umbral de sequía. Los diagramas evidencian diferencias en la frecuencia de ocurrencia de sequías entre las diferentes áreas homogéneas obtenidas. Los contrastes existentes son analizados en el apartado de discusión. Con el objetivo de visualizar de un modo más preciso la evolución del SPI en cada una de las series regionalizadas de sequía obtenidas, en la Figura 9 se compara la evolución de las mismas. 
Figura 4. Mapas de correlación espacial entre cada CPR y las series SPI12

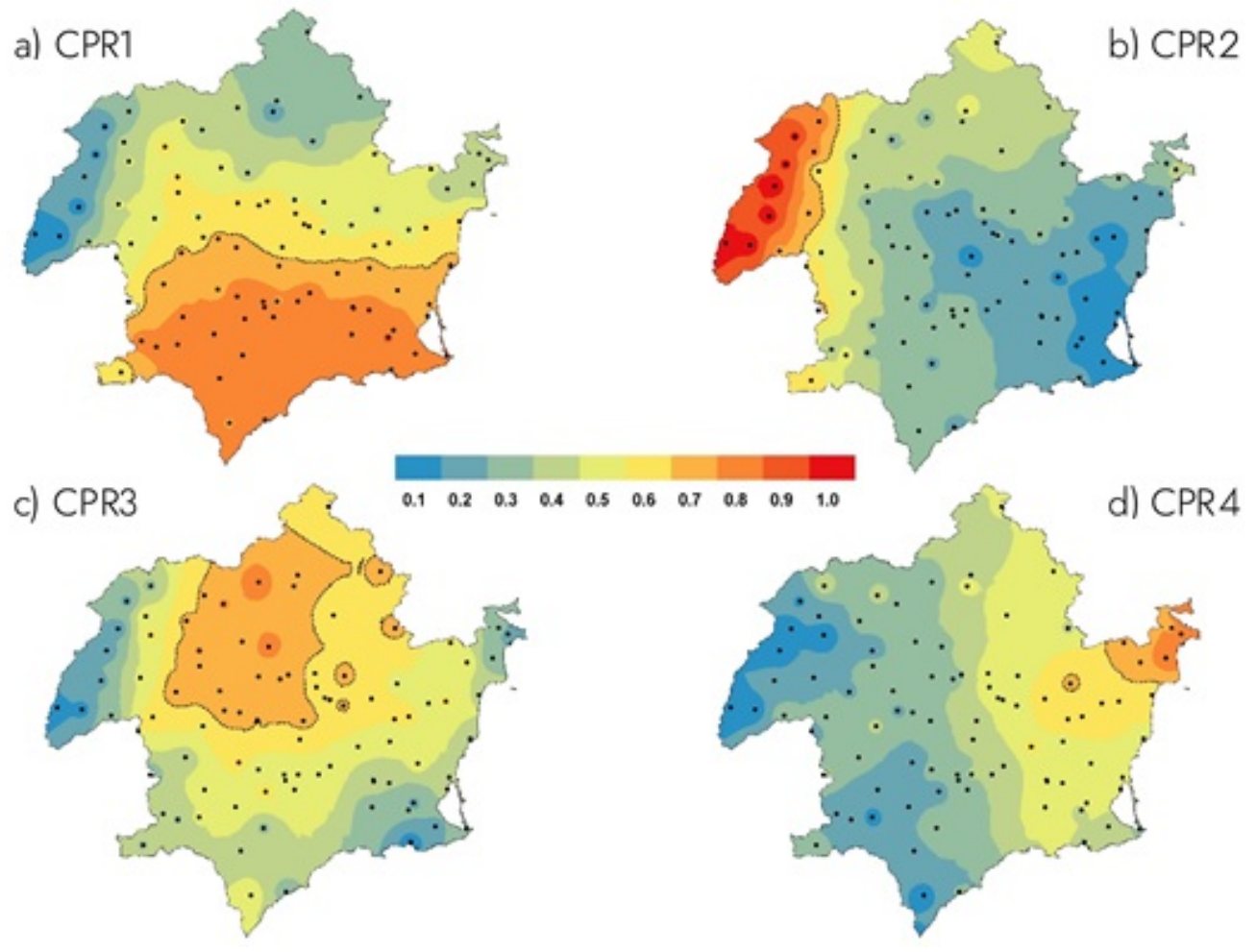

Fuente: elaboración propia

Figura 5. Evolución temporal de ocurrencia de sequías en el CPR1 mediante análisis con el método KORE

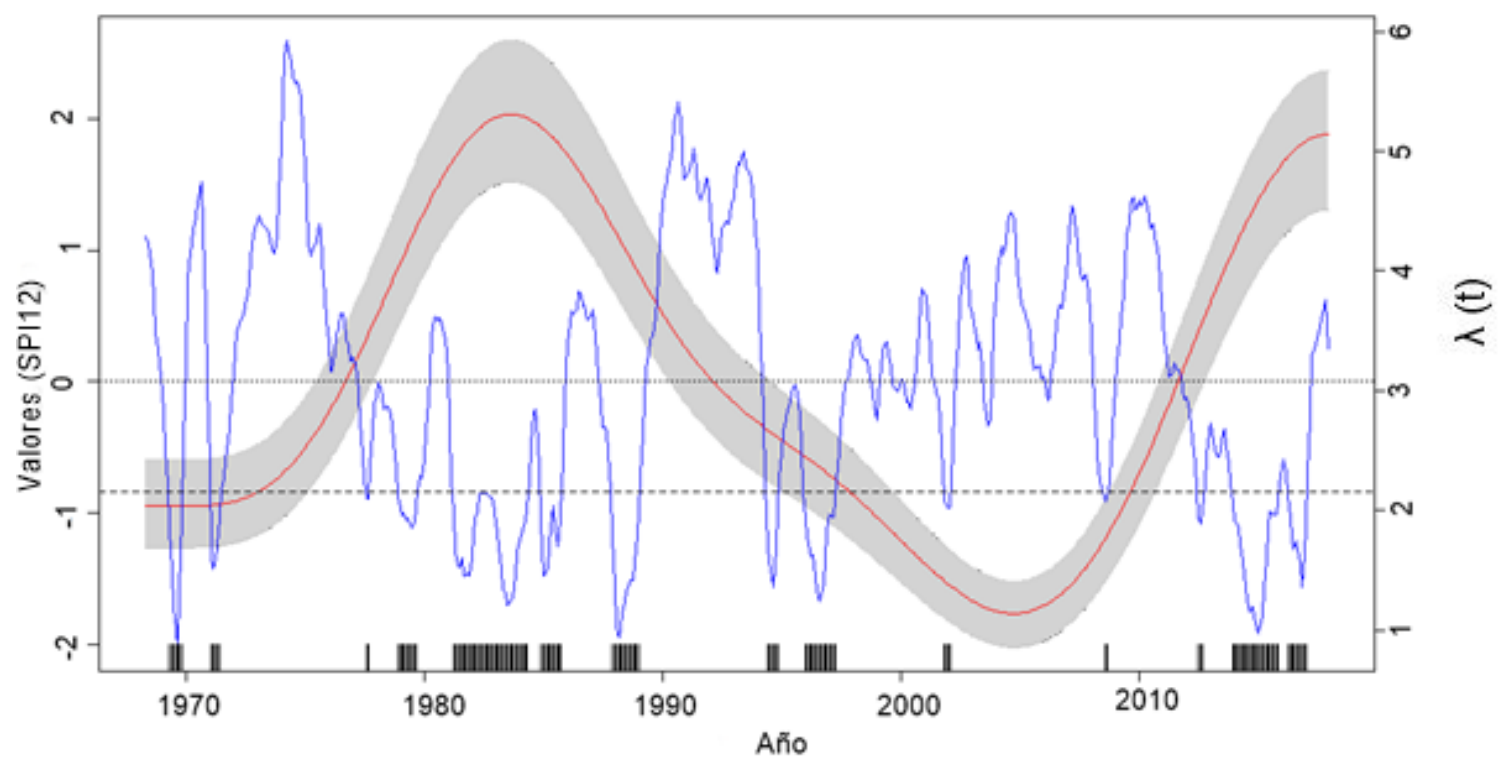

Nota: Las marcas negras verticales indican los puntos en el periodo temporal analizado por debajo del umbral de sequía adoptado $(-0,84)$

Fuente: elaboración propia 
Figura 6. Evolución temporal de ocurrencia de sequías

en el CPR2 mediante análisis con el método KORE

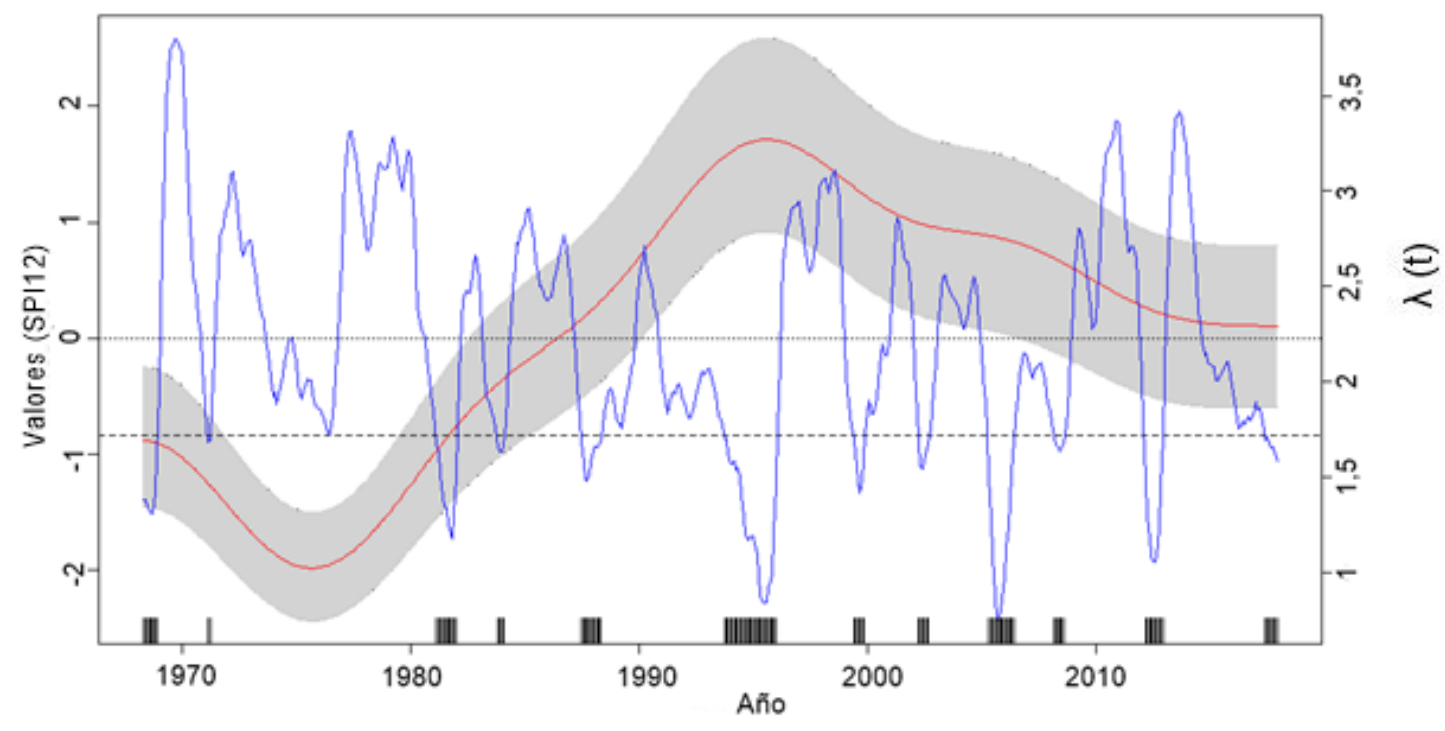

Nota: Las marcas negras verticales indican los puntos en el periodo temporal analizado por debajo del umbral de sequía adoptado $(-0,84)$

Fuente: elaboración propia

Figura 7. Evolución temporal de ocurrencia de sequías en el CPR3 mediante análisis con el método KORE

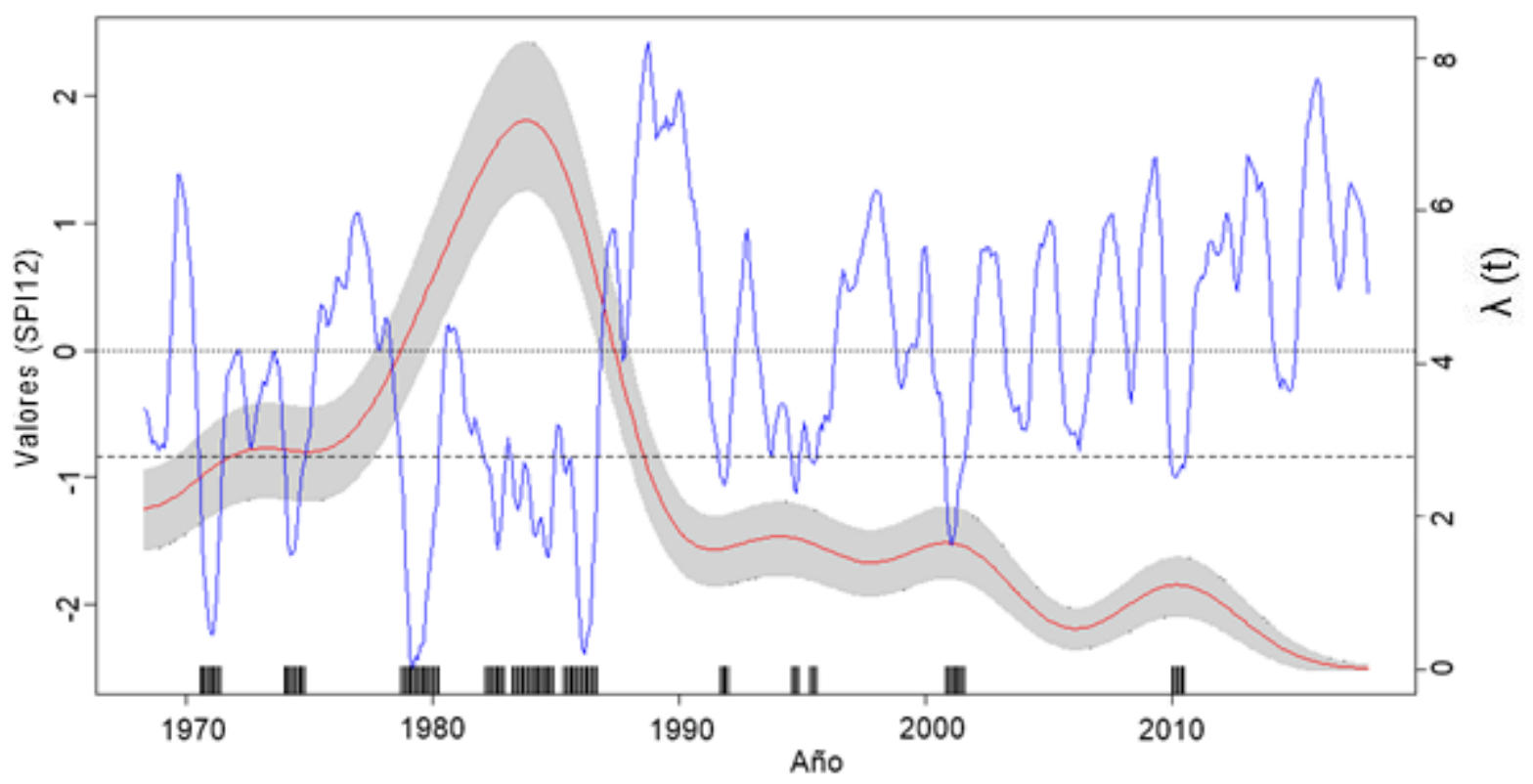

Nota: Las marcas negras verticales indican los puntos en el periodo temporal analizado por debajo del umbral de sequía adoptado $(-0,84)$

Fuente: elaboración propia 
Figura 8. Evolución temporal de ocurrencia de sequías en el CPR4 mediante análisis con el método KORE

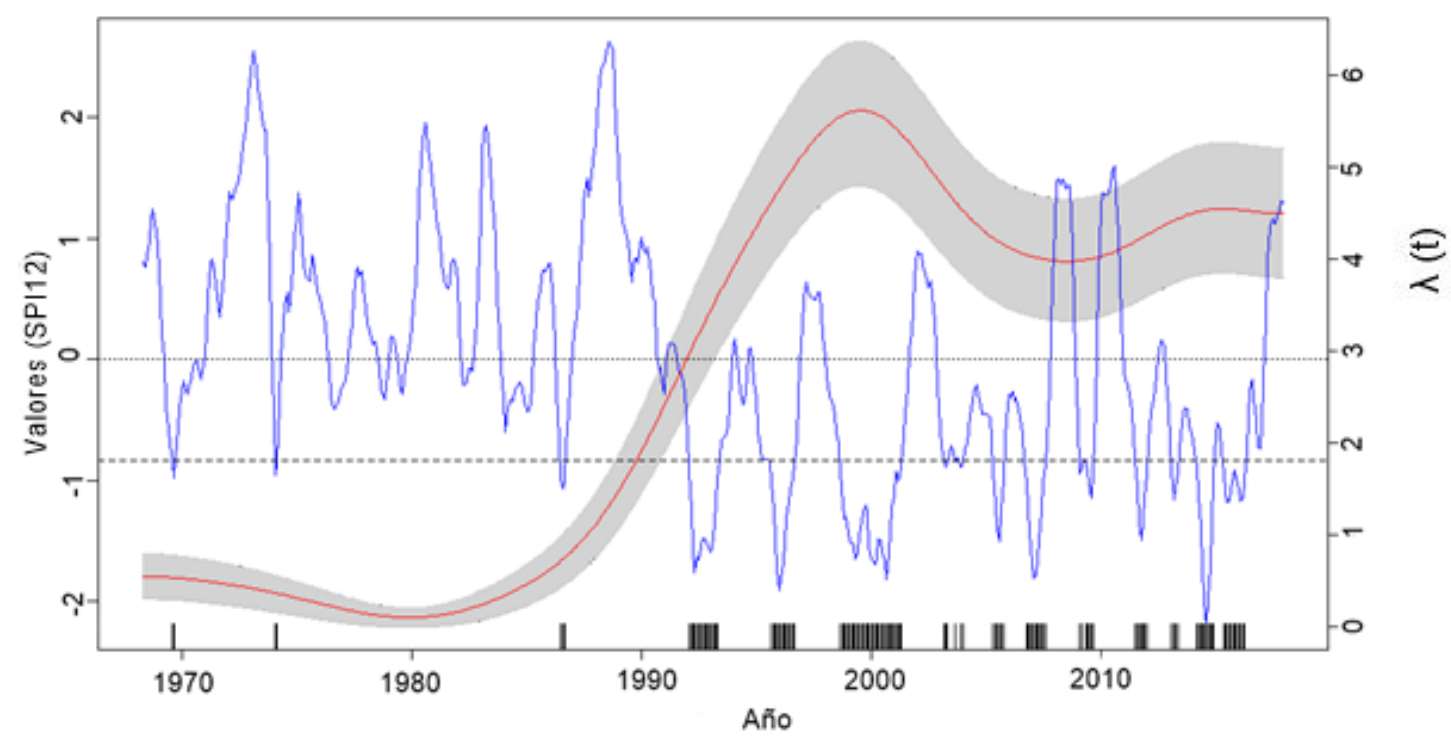

Nota: Las marcas negras verticales indican los puntos en el periodo temporal analizado por debajo del umbral de sequía adoptado $(-0,84)$

Fuente: elaboración propia 
Figura 9. Evolución del SPI (1968-2017)

en las series regionalizas (CPR) del SPI12 obtenidas


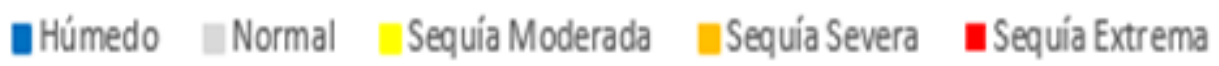

Fuente: elaboración propia

En la Figura 9, se observa como la CPR4 que se corresponde con la región más oriental, es la única que presenta una clara intensificación de los períodos de sequías en las tres últimas décadas. La CPR1 muestra un comportamiento parecido, con la diferencia que en las década de 
1970 y 1980 si se localizan varias secuencias pluviométricas secas. En la CPR3, se vislumbra como durante las tres últimas décadas, en esta serie regionalizada no se refleja ninguna sequía extrema. No obstante, en la figura 13 si se evidencia que parte del territorio de la RG3 si alcanzó la categoría de sequía extrema. La explicación radica en que las series que presentan una mayor correlación con esta CPR, no han alcanzado la categoría de sequía extrema desde la secuencia seca 1981-1986. Por último, en referencia a la CPR2 cabe destacar que es la que presenta un comportamiento más dispar, debido a la influencia de las sequías ibéricas.

En base a la delimitación realizada en los mapas de correlación espacial entre cada CPR y las series SPI12 (Figura 4) se ha llevado a cabo una zonificación por áreas homogéneas con una correlación igual o superior a 0,6 (Figura 10). Para tal fin, se ha asignado un área determinada a cada estación pluviométrica a partir del método de los polígonos de Thiessen (Thiessen, 1911). En la figura 10 se muestra como las regiones identificadas no se superponen y están espacialmente bien delimitadas cubriendo gran parte del territorio analizado. Tan solo 15 de las 92 estaciones analizadas presentan una correlación inferior a 0,6 con todas las CPR. Esto se corresponde con un total de 3097,4 km² (15,7\%). Las áreas por debajo de 0,6 de correlación con cada una de las CPR se constituyen como zonas de transición entre unas regiones y otras. Con el objetivo de que toda el área de estudio forme parte de una las regiones homogéneas, se han implementado otras técnicas de clúster (Ward's Euceldian y K-Means) para ayudar a asignar las 15 estaciones por debajo de 0,6 en una de las regiones homogéneas obtenidas con el análisis ACP. A través de este proceso las 15 estaciones anteriormente mencionadas han sido asignadas a una de las cuatro regiones homogéneas resultantes (Figura 10).

Las regiones homogéneas delimitadas en la Figura 10 (RG1 a RG4) representan superficies bastantes desiguales, que se distribuyen de la siguiente forma: RG1- 8481,4 km² $(43,1 \%)$ y 43 estaciones; RG2-2153 km² (10,9\%) y 10 estaciones; RG3-7832,5 km² (39,8\%) y 32 estaciones; RG4- $1230,7 \mathrm{~km}^{2}$ (6,2\%) y 7 estaciones. Desde un punto pluviométrico gran parte del área de estudio tiene una precipitación media anual inferior a los $400 \mathrm{l} / \mathrm{m}^{2}$ (figura 2), con la excepcionalidad de la zona noroccidental donde puntualmente se superan los $1000 \mathrm{l} / \mathrm{m}^{2}$. Por tanto, a excepción del extremo noroccidental (RG2) que se constituye con diferencia como la región más húmeda con una precipitación media anual de $636 \mathrm{l} / \mathrm{m}^{2}$, el resto de las regiones presentan una considerable uniformidad espacial en su precipitación media anual: RG1$307,7 \mathrm{l} / \mathrm{m}^{2}$; RG3- $348,5 \mathrm{l} / \mathrm{m}^{2}$; RG4- $287,3 \mathrm{l} / \mathrm{m}^{2}$. La precipitación media anual y estacional de cada región ha sido obtenida mediante la ponderación de las áreas de Thiessen. En la Tabla 3, 
se presenta la distribución estacional y anual de las precipitaciones por regiones en el periodo de referencia de 1981-2010, así como el coeficiente de variación anual para cada una de las regiones, con el objetivo de evaluar la irregularidad interanual de las precipitaciones. Se observa como la RG4 que es el territorio más árido, es el que presenta un coeficiente de variación más elevado, debido a elevada variabilidad interanual de las precipitaciones en este marco espacial.

Figura 10. Áreas homogéneas con valores del coeficiente de correlación superior a 0,6 entre cada CPR y las series SPI12 b) Regiones homogéneas definitivas

a)



b)



Leyenda: Las áreas en color gris representan las áreas con una correlación inferior a 0,6 con todas las CPR.

Fuente: elaboración propia

Tabla 3. Distribución anual y estacional de las precipitaciones por regiones y coeficiente de variación anual

\begin{tabular}{|c|c|c|c|c|c|c|}
\hline Región & Invierno & Primavera & Verano & Otoño & Anual & $\begin{array}{c}\text { Coeficiente } \\
\text { Variación } \\
\text { Anual }\end{array}$ \\
\hline RG1 & 83,4 & 89,9 & 30,2 & 104,2 & 307,7 & 29,7 \\
RG2 & 226,2 & 197,1 & 42,3 & 170,4 & 636,0 & 29,4 \\
RG3 & 94,0 & 102,4 & 36,7 & 115,4 & 348,5 & 27,5 \\
RG4 & 68,3 & 74,7 & 25,1 & 119,2 & 287,3 & 31,8 \\
\hline
\end{tabular}

Nota: Los datos se refieren al período de referencia 1981-2010.

Fuente: elaboración propia a partir de la base de datos reconstruida 


\subsection{Evolución temporal del área afectada por sequías}

Para cada una de las regiones homogéneas obtenidas (figura 10) se han identificado las áreas afectadas por las diferentes categorías de sequías (moderada, severa y extrema) asignadas por Agnew (2000). El área de cada región atribuida a cada categoría de sequía ha sido obtenida a partir de la aplicación de áreas acumulativas de los polígonos de Thiessen previamente calculados. Para cada región se presentan dos diagramas con un periodo temporal de 25 años (1968-1992 y 1993-2017) con la finalidad de facilitar su compresión y visualización. Los resultados obtenidos se reflejan en la figuras 11, 12, 13, 14. En las mismas se exhibe la superficie porcentual afectada por las diferentes categorías de sequía (son representadas con diferentes colores: moderada- azul, severa-naranja y extrema-gris). Con este proceso se han podido identificar de forma concisa los distintos patrones de sequía existentes en el territorio analizado, así como, la severidad de los distintos periodos de indigencia pluviométrica. De esta forma, es posible establecer pautas espaciales en el comportamiento de las sequías entre las distintas regiones.

Figura 11. Evolución temporal del porcentaje de área afectada por sequías con diferentes grados de severidad en la RG1



- MODERADA $\quad$ SEVERA EXTREMA

Fuente: elaboración propia 
Figura 12. Evolución temporal del porcentaje de área afectada por sequías con diferentes grados de severidad en la RG2

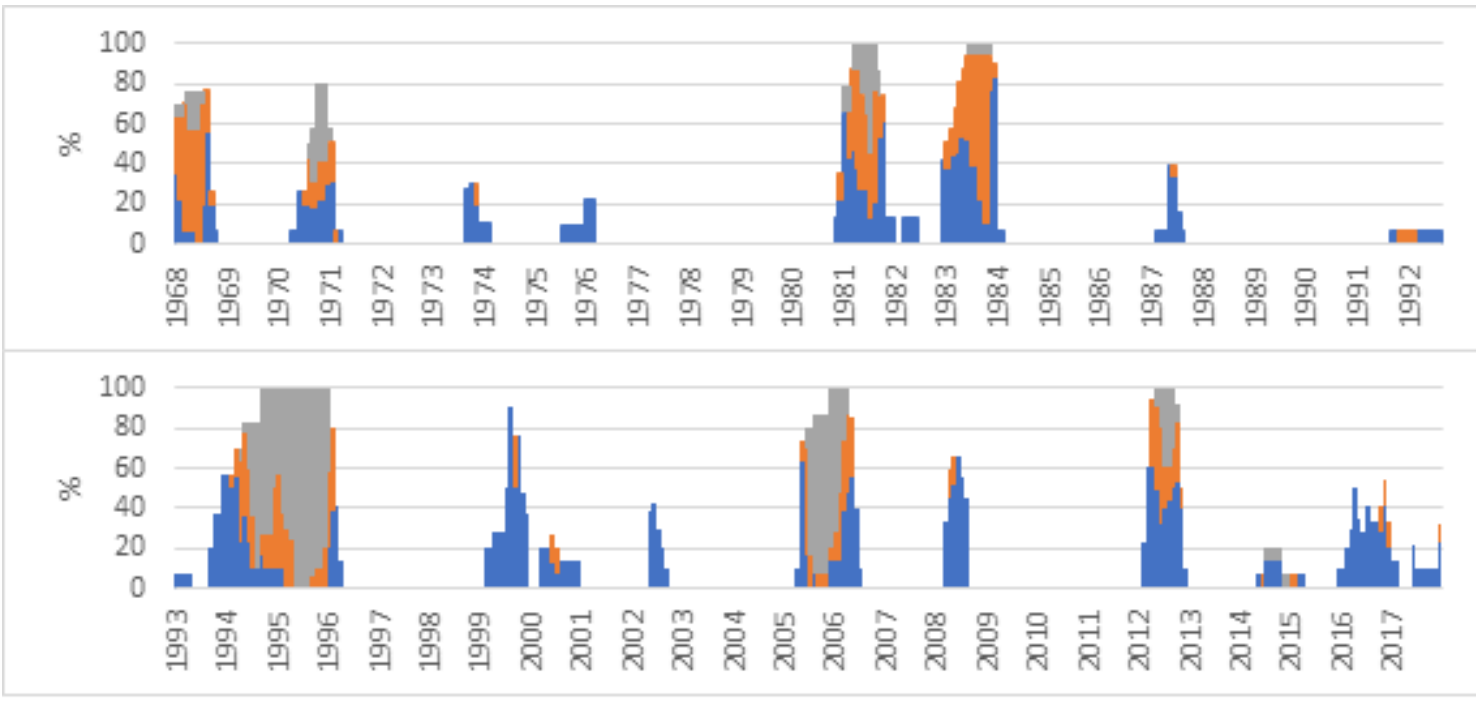

\section{- MODERADA SEVERA EXTREMA}

Fuente: elaboración propia

Figura 13. Evolución temporal del porcentaje de área afectada por sequías con diferentes grados de severidad en la RG3

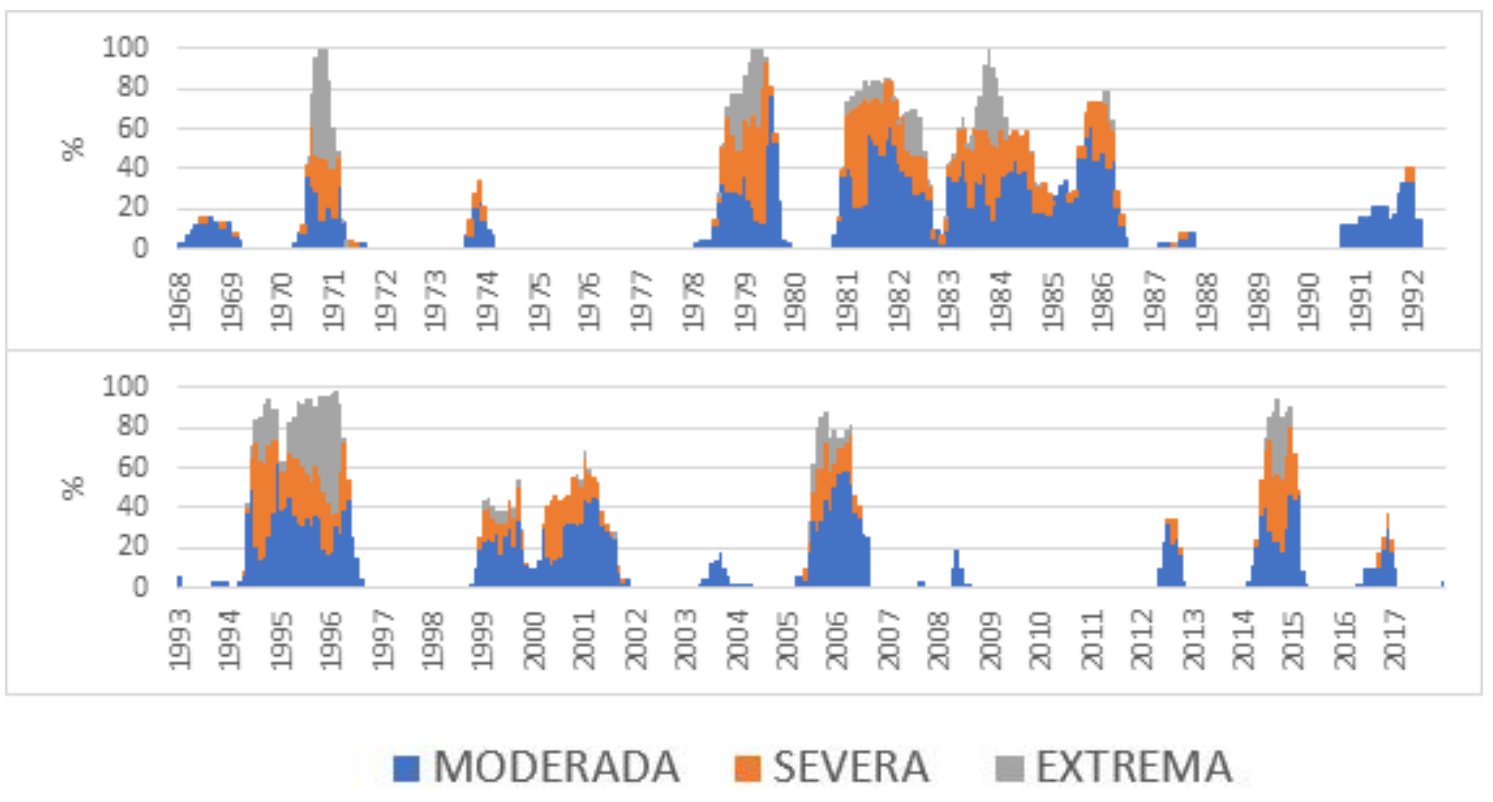

Fuente: elaboración propia 
Figura 14. Evolución temporal del porcentaje de área afectada por sequías con diferentes grados de severidad en la RG4

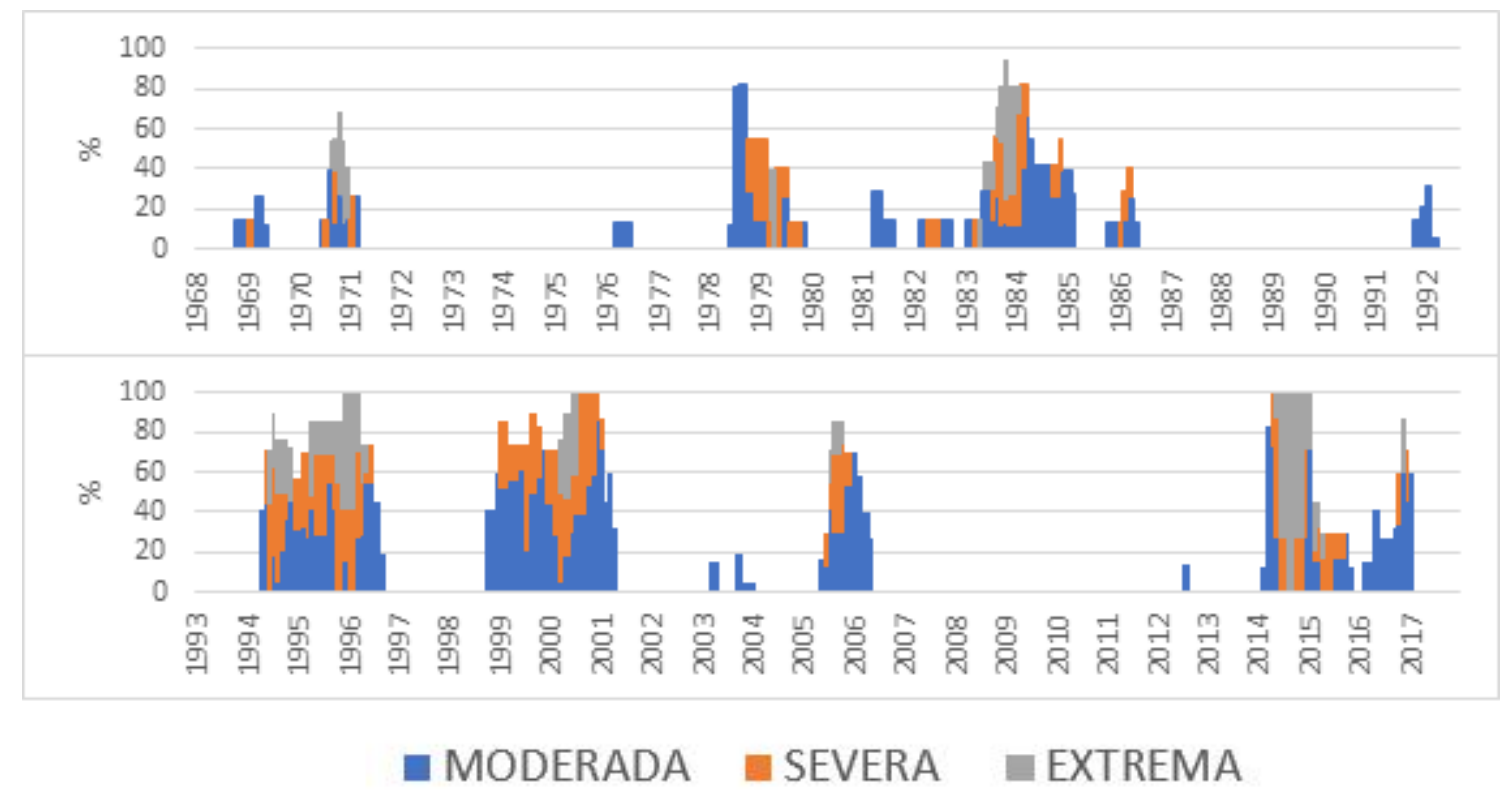

Fuente: elaboración propia

\section{Discusión}

Como ya ha sido mencionado, el ámbito territorial de la DHS es uno de los espacios más áridos de Europa, siendo la única demarcación hidrográfica de un total de 215 cuya precipitación anual es inferior a $450 \mathrm{l} / \mathrm{m}^{2}$. De este modo, este territorio posee una situación de escasez pluviométrica estructural lo que se deriva de un déficit hídrico igualmente estructural. El abastecimiento de agua potable a la mayor parte de la población se realiza a través de la MCT, procediendo sus recursos fundamentalmente de tres fuentes de abastecimiento: embalse del Taibilla, trasvase Tajo-Segura y desalinización. Paradójicamente, y a pesar del severo déficit hídrico existente en el territorio analizado, se llevó a cabo una importante reconversión de cultivos de secano a cultivos de regadío intensivo, auspiciado por las expectativas generadas ante la llegada en el año 1979 de las aguas procedentes de la cabecera del río Tajo. Este aumento descontrolado de los cultivos de regadío ha acabado incrementando déficit hídrico existente, que en la actualidad se estima en $400 \mathrm{hm}^{3}$ (suponiendo una aportación equivalente a la media de las aportaciones del trasvase Tajo-Segura durante el periodo 1980/81-2011/12).

Durante las dos últimas décadas se ha producido un aumento de la superficie de regadío en torno a un 11\% en la DHS (Morote \& Rico, 2018). De hecho, las actividades agrícolas junto con las actividades turísticas representan las principales actividades económicas del área de estudio, 
concentrándose sobre todo en las comarcas litorales donde se localiza también la mayor parte de la población. Desde el año 2004 con la implantación del programa A.G.U.A (Real DecretoLey 2/2004, convertido luego en la Ley 11/2005, de 22 de junio que modificaba la Ley 10/2001, del Plan Hidrológico Nacional) se ha ido aumentando poco a poco los aportes procedentes de la desalación en detrimento de los recursos procedentes del trasvase TajoSegura. El aumento del nivel de consideración de aguas excedentarias a partir del cual se pueden realizar trasvases desde la cabecera del Tajo (Ley 21/2013, de 9 de diciembre, de evaluación ambiental) desde $240 \mathrm{hm}^{3}$ hasta $400 \mathrm{hm}^{3}$ a partir del año 2018, junto a la estimada reducción drástica de los recursos en la cabecera del Tajo simulada por los escenarios de cambio climático (Pellicer \& Martínez, 2018), puede poner en riesgo la viabilidad futura de estos cultivos de regadío. Por tanto es necesario buscar nuevas fuentes de abastecimiento, que aseguren el suministro de agua en situaciones futuras de prolongada sequía. En Morote y Rico (2018), se analiza concienzudamente el impulso de los recursos hídricos procedentes de la desalinización.

Ante estos escenarios, los estudios de regionalización de sequías resultan de vital importancia con el objetivo de monitorizar diferentes regímenes de sequía y su distribución espacial. En este trabajo se ha realizado una regionalización de sequías a partir del análisis ACP. A través de la rotación de los componentes principales (CPR) se han seleccionado los cuatro primeros componentes, ya que mediante el mapeo de los valores de las correlaciones entre las SPI12 y las series CPR, se observó que estos componentes cubrían gran parte del área de estudio sin superponerse (Figura 4).

Por otra parte, Olcina (2001b) expone que en España es posible distinguir entre cuatro tipos básicos de sequía: sequías cantábricas, ibéricas, surestinas y canarias. El territorio analizado es afectado por dos de ellas: ibéricas y surestinas. Las sequías ibéricas tienen carácter coyuntural y afectan a la práctica totalidad de la península ibérica. Este tipo de secuencias pluviométricas secas suele desencadenarse por un ciclo de prevalencia de la fase positiva del patrón de teleconexión North Atlantic Oscillation (NAO), que provoca que las borrascas circulen por latitudes más septentrionales de Europa, reduciéndose por tanto la incidencia de frentes atlánticos sobre la península ibérica (Vázquez,1999). Por el contrario, las sequías surestinas, afectan casi en exclusividad al ámbito del sureste ibérico, y se originan fundamentalmente por una fase negativa de la NAO que favorece el desplazamiento hacia el sur de los frentes atlánticos, con el correspondiente aumento de las precipitaciones en gran parte del territorio peninsular (Gallego et al., 2005). En gran parte del área de estudio, localizada a sotavento de 
dicho flujo húmedo atlántico, los frentes llegan bastantes degradados debido a la acción de barrera orográfica (efecto Foehn) de relieves como la Sierra de Segura (Capel, 1983).

Por su parte, los patrones espacio-temporales de las sequías han sido estudiados mediante el análisis KORE para estimar la frecuencia de ocurrencia de sequías, y a través del diagnóstico de la evolución temporal del porcentaje de área afectada por sequías con diferentes grados de severidad para cada una de las cuatro regiones homogéneas obtenidas. Con ello se han podido monitorizar diferentes regímenes de sequía y su distribución espacial. En primer lugar cabe destacar que los ciclos de sequía más importantes tienden a afectar a todo el área de estudio con diferentes grados de severidad. Estas secuencias secas son: 1970-1971; 1981-1986, 1993-1996, 1999-2001, 2005-2006 y 2014-2015.

La RG2 situada en el extremo noroccidental es la que presenta patrones de sequía más diferenciados debido a la marcada influencia atlántica de esta zona, dependiendo sus precipitaciones en gran parte de los frentes atlánticos sobre todo durante los equinoccios e invierno. Por dicho motivo se configura como la única región donde las sequías ibéricas tienen una gran repercusión. En esta región, las consecuencias negativas de las secuencias secas transcienden a toda el área de estudio, debido a que en ella se originan la mayor parte de los recursos hídricos (cabeceras de los ríos Segura y Mundo). Por tanto, los periodos secos en este territorio generan intensas sequías hidrológicas que pueden ocasionar importantes problemas de abastecimiento de agua. Además en esta área se localiza el embalse del Taibilla, una de las principales fuentes de abastecimiento de la MCT y punto de origen de esta infraestructura.

Por otro lado, es muy importante resaltar que a todos estos aspectos hay que añadir que tanto la cabecera del río Tajo (origen del Trasvase Tajo-Segura) como la cabecera del río Segura se ven afectadas por los mismos mecanismos generados de secuencias secas, por lo que durante los eventos de sequía ibérica los trasvases de agua desde la cabecera del Tajo son bastante inviables, más si cabe con el cambio de reglas de explotación anteriormente mencionado. Esto lleva consigo una drástica sequía socioeconómica sobre todo en las comarcas litorales y áreas metropolitanas de Murcia y Alicante donde se concentran gran parte de la población y de las actividades económicas, que son muy dependientes de los recursos trasvasados sobre todo en el caso de los cultivos intensivos de regadío. Con el análisis KORE se observa cierto incremento en la frecuencia de ocurrencia de sequías en el periodo analizado en esta región, lo cual se corrobora en el gráfico de evolución de área afectada por las sequías. Se destacan los ciclos secos de 1993-1996 y 2005-2006, ya que casi todo el área de esta región se vio afectada 
por sequías extremas. Este hecho es corroborado en otros estudios realizados en el ámbito peninsular donde se observa una intensificación de los periodos de sequía desde la segunda mitad del siglo XX (Sousa et al., 2011; González et al., 2018).

En cambio las RG1 y RG4 presentan una marcada influencia mediterránea en su régimen pluviométrico. Además cabe resaltar la elevada torrencialidad de las precipitaciones de ambas áreas debido a la compleja orografía y la cercanía al mar Mediterráneo. Como ya ha sido mencionado, en el litoral de ambas zonas se localizan los récords de precipitación máxima diaria, que en muchos casos superan o igualan los registros de precipitación media anual. Estos episodios de lluvias torrenciales están ocasionados en la mayor parte de los casos por Depresiones Aisladas en Niveles Altos (DANA) que se ubican en torno al Golfo Cádiz o Mar de Alborán favoreciendo la entrada de vientos húmedos de largo recorrido marítimo. Sin embargo, los episodios adversos de precipitaciones en el área mediterránea pueden estar también ligados a otro tipo de procesos, donde la compleja orografía litoral de las cordilleras béticas juega un papel fundamental de disparo orográfico (Conesa \& Alonso, 2006). En definitiva la sucesión de largos periodos de sequía con intensos episodios de lluvias torrenciales es una de sus principales características climáticas de estas regiones litorales y prelitorales.

Por tanto en ambas regiones las sequías surestinas tienen una marcada importancia, sobre todo en la RG4. No obstante los mecanismos desencadenantes de las sequías ibéricas también afectan a ambas regiones aunque con menor severidad con otras zonas de la península ibérica. Además hay que resaltar que en el sureste peninsular se prolongan los efectos de las sequías ibéricas (pasando a ser surestinas), puesto que estas suelen finalizar debido a llegada sucesiva de frentes atlánticos que provocan precipitaciones en la mayor parte de la península ibérica (Olcina, 2001b). Las configuraciones sinópticas que dan lugar a las secuencias secas surestinas originan sequías agrícolas muy intensas, con importantes pérdidas en los cultivos de secano. La implementación en las últimas décadas de actividades como los cultivos de regadío o el desarrollo de actividades turísticas ha provocado que estos usos del suelo excedan de los recursos de agua endógenos de estas áreas. Por ello, la dependencia de recursos hídricos junto a la concentración de la mayor parte de las reservas hídricas en la cabecera del Segura provoca que durante secuencias secas ibéricas, las sequías hidrológicas sean muy intensas. Esto da lugar, a importantes impactos sociales, económicos y medioambientales (sequía socioeconómica) que son especialmente destacados en las dos regiones citadas donde se concentra la mayor parte de la población. 
En estas dos regiones se observa un aumento en la frecuencia de ocurrencia de sequías, siendo este especialmente significativo en la RG4, debido a la reducida presencia de sequías en las dos primeras décadas del periodo estudiado. En cambio en la RG1 la sequía de 1981-1986 fue la de mayor duración y magnitud de todo el periodo. Esta sequía también se refleja en la RG3. Esta secuencia seca también fue especialmente severa en el conjunto del suroeste europeo (Spinoni et al., 2015). En las últimas décadas las secuencias secas más destacadas han sido 1993-1997, 1999-2001 y 2014-2015, siendo estas dos últimas sequías surestinas. La sequía de 2014-2015 es una de las más severas del periodo estudiado, resultando afectado en torno a un $80 \%$ de estos territorios por una sequía extrema. Esta sequía se originó tras un año hidrológico (2012-2013), muy húmedo en toda la península ibérica excepto en el sureste.

Por último, la RG3 ubicada en gran parte de la mitad septentrional del área de estudio, es la única región en la que se observa una disminución en la frecuencia de ocurrencia de sequías en las últimas décadas. Este hecho se corrobora con la evolución del área afectada por sequías dado que desde la sequía de 1993-1996 no se ha visto afectado en ningún el 100\% del territorio por sequías, siendo la presencia de sequía extrema muy reducida en las dos últimas décadas. Se trata de una zona de transición entre la influencia de los frentes atlánticos y las precipitaciones de origen mediterráneo. En Miró et al. (2018) se observa un ligero incremento en la precipitación media anual en gran parte de esta región durante el periodo 1955-2016. Esta tendencia ascendente se refleja sustancialmente en las precipitaciones de origen mediterráneo y de origen convectivo (sobre en el periodo de octubre a abril). En este estudio se expone que esta área es prácticamente el único territorio de la DHS donde se observan estos incrementos. Entre las secuencias secas más intensas caben destacar los periodos 1981-1986 y 1993-1996.

La regionalización de sequías obtenida en el presente trabajo podría ser complementaria a las unidades territoriales de sequía y escasez (UTS y UTE), establecidas en el vigente Plan Especial de Sequía de la Demarcación Hidrográfica del Segura (CHS, 2018). Precisamente se establecen un total de cuatro unidades territoriales cuya delimitación espacial se asemeja en parte a la obtenida en el presente trabajo, sobre todo en la zona de la cabecera del Segura. No obstante, en esta delimitación espacial también entra en juego el Trasvase Tajo-Segura. Asimismo, los resultados obtenidos podrían incluirse en un hipotético plan de protección civil frente a las sequías. En Aparicio (2007) se analiza la necesidad de la elaboración de estos planes, como medida de prevención y mitigación de los efectos negativos de las sequías. 


\section{Conclusión}

Las sequías son el riesgo de origen climático que mayor número de afectados y repercusiones económicas, sociales y territoriales generan en los espacios geográficos donde se desarrollan. En este trabajo se realiza una caracterización exhaustiva de las sequías en el ámbito territorial de la DHS y MCT, ubicadas en el sureste de la península ibérica, que se constituye como el área más árida del continente europeo. La DHS es la única demarcación del continente europeo con una precipitación media inferior a $450 \mathrm{l} / \mathrm{m}^{2}$, lo cual genera un importante déficit pluviométrico estructural y un consecuente déficit hídrico notable que se ve acentuado por la implementación de usos del suelo que exceden de los recursos de agua endógenos.

El principal objetivo de este trabajo ha sido la realización de una regionalización de sequías a partir del índice SPI con la finalidad de monitorizar diferentes patrones de sequía y su distribución espacial. La misma ha sido elaborada mediante un análisis ACP. Para el estudio se han utilizado un total de 92 series de precipitación mensual, siendo 1968-2017 el periodo temporal seleccionado. A través de la rotación de los componentes principales (CPR) se han seleccionado los cuatro primeros componentes.

De este modo, se han delimitado cuatro regiones homogéneas (Figura 10) cuya distribución espacial es bastante razonable desde un punto de vista hidrológico, climático y orográfico. Los ciclos de sequía de mayor gravedad tienden a afectar a toda el área de estudio con diferentes grados de severidad. Estas secuencias secas son: 1970-1971; 1981-1986, 1993-1996, $1999-$ 2001, 2005-2006 y 2014-2015. La RG2 situada en el extremo noroccidental (cabeceras de los ríos Segura y Mundo) es la que presenta patrones de sequía más diferenciados debido a la marcada influencia atlántica de esta área. En esta región las sequías ibéricas tienen una gran incidencia provocando intensas sequías hidrológicas que repercuten en toda el área estudio, debido a que en ella se originan la mayor parte de los recursos hídricos. Además, se observa un ligero aumento de la frecuencia de ocurrencia de sequías, así como del área afectada por las mismas.

Por el contrario, las RG4 y RG1 situadas en la mitad meridional y extremo oriental respectivamente, presentan una marcada influencia mediterránea en su régimen de precipitaciones. Se observa un incremento en la frecuencia de ocurrencia de sequías y en el área afectada por las mismas, siendo este especialmente significativo en la RG4. La dependencia de recursos hídricos debido a la implementación de usos del suelo que exceden de los recursos de agua endógenos provoca que durante las secuencias secas ibéricas las sequías hidrológicas 
muy intensas. Esto genera importantes impactos sociales, económicos y medioambientales en estas regiones litorales y prelitorales (sequía socioeconómica). Los escenarios de cambio climático prevén una drástica reducción de los recursos hídricos en la cabecera del Tajo, por lo que se tienen que implementar una serie de medidas encaminadas a la reducción de la vulnerabilidad y exposición de la población frente a las sequías. La desalinización puede jugar en este aspecto un papel fundamental. Finalmente, la RG3 situada en gran parte de la mitad septentrional del área de estudio es la única donde se observa una reducción de la frecuencia de ocurrencia por las sequías, así como del área afectada por sequías extremas.

En definitiva, en el presente estudio el análisis ACP ha resultado un método eficaz para la realización de una regionalización de sequías, tal y como se ha demostrado en otras investigaciones. Las regiones obtenidas podrían ser complementarias a las unidades territoriales de sequía y escasez, establecidas en el Plan Especial de Sequías de la Demarcación Hidrográfica del Segura.

Por último, como propuestas de trabajo futuro se pueden destacar las siguientes: a) Aplicación del mismo marco metodológico pero con la aplicación del SPEl (Vicente et al., 2010) para estudiar el impacto del incremento las temperaturas sobre las sequías; b) Analizar en profundidad en las tendencias y cambios en los parámetros de duración y magnitud de sequías que complementen los resultados y conclusiones obtenidos en este estudio; c) Implementación de un análisis de alerta temprana y probabilidad de ocurrencia de sequías mediante el uso de cópulas.

Agradecimientos: Esta investigación forma parte del programa de ayudas para la Formación de Profesorado Universitario (FPU), bajo cuyo auspicio el autor principal de esta investigación ha disfrutado de un contrato predoctoral [MECD-FPU15/01792].

Declaración responsable: Las/os autoras/es declaran que no existe ningún conflicto de interés con relación a la publicación de este artículo. Las tareas se han distribuido de la siguiente manera: el artículo ha sido coordinado por V. Ruiz. F. Belmonte y R. García han participado en los apartados de Introducción y Discusión. M.M. Portela, L.A. Espinosa han elaborado la metodología y resultados. La elaboración y procesamiento de datos estuvo a cargo de M. Ruiz. 


\section{Bibliografía}

Abdi, H., \& Williams, L. J. (2010). Principal component analysis. Wiley interdisciplinary reviews: computational statistics, 2(4), 433-459.

Agnew, C.T. (2000). Using the SPI to identify drought. Drought Network News, 12, 6-12.

Álvarez Rodríguez, J. (2004). Regionalización y caracterización de sequías en Europa. Revista Digital del Cedex, (137), $143 . \quad$ Retrieved from http://ingenieriacivil.cedex.es/index.php/ingenieria-civil/article/view/2161

Aparicio Florido, J. A. (2007). El riesgo de sequía y su inclusión en los planes de protección civil. Boletín de la Asociación Geógrafos Españoles, (44), 95-116. Retrieved from hitps://bage.age-geografia.es/ojs//index. php/bage/article/view/611

Bartolomeu, S., Carvalho, M. J., Marta-Almeida, M., Melo-Gonçalves, P., \& Rocha, A. (2016). Recent trends of extreme precipitation indices in the Iberian Peninsula using observations and WRF model results. Physics and Chemistry of the Earth, (94), 1021. https://doi.org/10.1016/j.CPe.2016

Benhamrouche, A., \& Martín-Vide, J. (2011). Distribución espacial de la concentración diaria de la precipitación en la provincia de Alicante. Investigaciones Geográficas, (56), 113129. hittps://doi.org/10.14198/ingeo2011.56.06

Camarillo Naranjo, J. M. (2012). Diseño, metodología y modelo de datos para el cálculo, seguimiento y predicción del índice estandarizado de sequía pluviométrica en Andalucía (Doctoral dissertation, Universidad de Sevilla, Spain). Retrieved from hitps://idus.us.es/handle/11441/24224

Capel Molina, J.J. (1983). Distribución de la lluvia en el sureste español. Periodo 1951-1989. Boletín del Instituto de Estudios Almerienses, 3, 27-36.

Castells, F., Laguna, P., Sörnmo, L., Bollmann, A., \& Roig, J. M. (2007). Principal component analysis in ECG signal processing. EURASIP Journal on Advances in Signal Processing, 2007(1), 074580. https://doi.org/10.1155/2007/74580

Charre, J. (1977). A propos de sécheresse. Revue de Géographie de Lyon, 52(2), 215226. https://doi.org/10.3406/geoca.1977.1204

CHS (2018). Revisión del Plan Especial de Sequía de la Demarcación Hidrográfica del Segura. Confederación Hidrográfica del Segura, Ministerio para la Transición Ecológica. 
Conesa García, C. (2006): Las formas del relieve. En C. Conesa (Ed.), El medio físico de la Región de Murcia (pp. 47-94). Murcia: Servicios de Publicaciones de la Universidad de Murcia.

Conesa García, C., \& Alonso Sarría, F. (2006). El clima de la Región de Murcia. In C. Conesa, (Ed.), El medio físico de la Región de Murcia (pp. 95-128). Murcia: Servicios de Publicaciones de la Universidad de Murcia.

De Luis, M., González-Hidalgo, J. C., Longares, L. A., \& Stepánek, P. (2009). Seasonal precipitation trends in the Mediterranean Iberian Peninsula in second half of 20th century. International Journal of Climatology, (29), 1312-1323. https://doi.org/10.1002/joc. 1778

Del Moral, L., Hernández-Mora, N., De Stefano, L., Paneque, P., Vargas, J., Brufao, P., OlcinaCantos, J., \& Martínez-Fernández, J. (2017). Acerca del Real Decreto Ley 10/2017, de 9 de junio, por el que se adoptan medidas urgentes para paliar los efectos producidos por la sequía en determinadas cuencas hidrográficas y se modifica el texto refundido de la Ley de Aguas, aprobado por el Real Decreto Legislativo 1/2001, de 20 de julio. Fundación Cultura del Agua.

Diggle, P. (1985). A kernel method for smoothing point process data. Journal of the Royal Statistical Society: Series C (Applied Statistics), 34(2), 138-147.

Dillan, W., \& Goldstein, M. (1986). Multivariate Analysis: Method and Applications (pp. 85-87). In Technometrics, (28). https://doi.org/10.1080/00401706.1986.10488103

Domínguez-Castro, F., Vicente-Serrano, S. M., Tomás-Burguera, M., Peña-Gallardo, M., Beguería, S., El Kenawy, A., Luna, Y., \& Morata, A. (2019). High spatial resolution climatology of drought events for Spain: 1961-2014. International Journal of Climatology, 39(13), 50465062. https://doi.org/10.1002/joc.6126

Espinosa, L.A., Portela, M.M., \& Rodrigues, R. (2019). Spatio-temporal variability of droughts over past 80 years in Madeira Island. Journal of Hydorology: Regional Studies, 25, 100623. https://doi.org/10.1016/j.ejrh.2019.100623

Fleig, A. K., Tallaksen, L. M., Hisdal, H., \& Demuth, S. (2006). A global evaluation of streamflow drought characteristics. Hydrology and Earth System Sciences Discussions, 10(4), 535-552. Retrieved from www. hydrol-earth-syst-sci.net/10/535/2006/

Font Tullot, I. (1988). La singularidad persistencia de situaciones anticiclónicas sobre la península ibérica durante el cuatrimestre noviembre 1988-febrero 1989. Paralelo, 37(11), 75-84. 
Ford, T. W., McRoberts, D. B., Quiring, S. M., \& Hall, R. E. (2015). On the utility of in situ soil moisture observations for flash drought early warning in Oklahoma, USA. Geophysical Research Letters, 42(22), 9790-9798. https://doi.org/10.1002/2015GL066600

Gallego, M.C., García, J.A, \& Vaquero, J.M. (2005): The NAO signal in daily rainfall series over the Iberian Peninsula. Climate Research, 29(2), 103-109.

García-Galiano, S.G., Baille, A., González, M.M., Martínez, V., Urrea, M., Hernández, Z., Nortes, P., \& Tanguy, M. (2007). Desarrollo y aplicación de indicadores de alerta temprana frente a sequías a escala regional desde MODIS. In Teledetección: Hacía un entendimiento de la dinámica global y regional (pp. 223-229). XII Congreso de la Asociación Española de Teledetección

García-Marín, R. (2008). Riesgo de sequía y vulnerabilidad socioeconómica en la Cuenca del Guadalentín (Doctoral dissertation, Universidad de Murcia, Spain). Retrieved from https://digitum.um.es/digitum/handle/10201/3566

Garrido-Abenza, R., Palenzuela-Cruz, J. E., \& Bañon-Pelegrín, L. M. (2014). Atlas climático de la Región de Murcia. Agencia Estatal de Meteorología.

Gallego, M. C., Trigo, R. M., Vaquero, J. M., Brunet, M., García, J. A., Sigró, J., \& Valente, M. A. (2011). Trends in frequency indices of daily precipitation over the Iberian Peninsula during the last century. Journal of Geophysical Research Atmospheres,

116(2). https://doi.org/10.1029/2010JD014255

Gil Olcina, A. (2007). Mediterraneidad y subtropicalidad climáticas. Boletín de la Asociación de Geógrafos Españoles, (44), 53-68. Retrieved from https://bage.agegeografia.es/ojs/index.php/bage/article/view/609

González-Hidalgo, J. C., Brunetti, M., \& de Luis, M. (2011). A new tool for monthly precipitation analysis in Spain: MOPREDAS database (monthly precipitation trends December 1945-November 2005). International Journal of Climatology, 31(5), 715-731. https://doi.org/10.1002/joc.2115

González-Hidalgo, J. C., Vicente-Serrano, S. M., Peña-Angulo, D., Salinas, C., Tomas-Burguera, M., \& Beguería, S. (2018). High-resolution spatio-temporal analyses of drought episodes in the western Mediterranean basin (Spanish mainland, Iberian Peninsula). Acta Geophysica, 66(3), 381-392. https://doi.org/10.1007/s11600-018-0138-x

Hair, J. F., Black, W. C., Babin, B. J., Anderson, R. E., \& Tatham, R. L. (1998). Multivariate data analysis, vol. 5, Prentice Hall. 
He, Y., Ye, J., \& Yang, X. (2015). Analysis of the spatio-temporal patterns of dry and wet conditions in the Huai River Basin using the standardized precipitation index. Atmospheric Research, 166, 120-128. https://doi.org/10.1016/j.atmosres.2015.06.022

Lehner, B., Döll, P., Alcamo, J., Henrichs, T., \& Kaspar, F. (2006). Estimating the impact of global change on flood and drought risks in Europe: a continental, integrated analysis. Climatic Change, 75(3), 273-299. https://doi.org/10.1007/s10584-006-6338-4

López-Moreno, J. I., Vicente-Serrano, S. M., Beguería, S., García-Ruiz, J. M., Portela, M. M., \& Almeida, A. B. (2009). Dam effects on droughts magnitude and duration in a transboundary basin: The Lower River Tagus, Spain and Portugal. Water Resources Research, 45(2). https://doi.org/10.1029/2008WR007198

Marcos Valiente, O. (2001). Sequía: definiciones, tipologías y métodos de cuantificación. Investigaciones Geográficas, (26), 59-80. https://doi.org/10.14198/ingeo2001.26.06

Martin-Vide, J. (2004). Spatial distribution of a daily precipitation concentration index in peninsular Spain. International Journal of Climatology, 24(8), 959971. https://doi.org/10.1002/joc. 1030

McKee, T. B., Doesken, N. J., \& Kleist, J. (1993). The relationship of drought frequency and duration to time scales. In Proceedings of the 8th Conference on Applied Climatology, 17(22), 179-183.

Miró, J. J., Estrela, M. J., Caselles, V., \& Gómez, I. (2018). Spatial and temporal rainfall changes in the Júcar and Segura basins (1955-2016): Fine-scale trends. International Journal of Climatology, 38(13), 4699-4722. https://doi.org/10.1002/joc.5689

Mishra, A. K., \& Singh, V. P. (2011). Drought modeling - A review. Journal of Hydrology, 403, 157-175. https://doi.org/10.1016/j.jhydrol.2011.03.049

Morales-Gil, A., Olcina-Cantos, J., \& Rico-Amorós, A. M. (1999). Diferentes perceCPiones de la sequía en España: adaptación, catastrofismo e intentos de corrección. Investigaciones Geográficas, (23), 5-46. https://doi.org/10.14198/ingeo2000.23.06

Morote Seguido, A. F., \& Rico Amorós, A. M. (2018). Perspectivas de funcionamiento del trasvase Tajo-Segura (España): efectos de las nuevas reglas de explotación e impulso de la desalinización como recurso sustitutivo. Boletín de la Asociación de Geógrafos Españoles, 79, 2754, 1-43. http://dx.doi.org/10.21138/bage.2754 
Morote-Seguido, A.F. (2019). Galicia, ¿Territorio adaptado a la sequía? Cuadernos Geográficos, 58(2), 6-33. https://doi.org/10.30827/CUADGEO.V58I2.7627

Mudelsee, M. (2011). The bootstrap in climate risk analysis. In Extremis: Disruptive Events and Trends in Climate and Hydrology (pp. 44-58). https://doi.org/10.1007/978-3-642-14863-7_2 Mudelsee, M., Bö Rngen, M., Tetzlaff, G., \& Grü Newald, U. (2003). No upward trends in the occurrence of extreme floods in central Europe. Nature, 425(6954), 166169. https://doi.org/10.1038/nature01928

Noguera, I., Domínguez-Castro, F., \& Vicente-Serrano, S. M. (2020). Characteristics and trends of flash droughts in Spain, 1961-2018. Annals of the New York Academy of Sciences, 1472(1), 155-172. https://doi.org/10.1111/nyas. 14365

Olcina Cantos, J. (2001a). Situaciones atmosféricas y tipos de tiempo. In J. Martín Vide \& J. Olcina Cantos (Eds.), Clima y tiempos en España (pp. 81-102). Madrid: Alianza editorial.

Olcina-Cantos, J. (2001b). Tipologías de sequía en España. Ería: Revista cuatrimestral de geografía, 56, 201-207. Retrieved from http://rua.ua.es/dspace/handle/10045/23004

Olcina-Cantos, J. (2006). ¿Riesgos naturales? I. Sequías e inundaciones. Barcelona: Davinci Continental.

Palmer, W.C. (1965). Meteorological drought (Reseach Paper, 45). Washington DC: US Department of Commerce Weather Bureau.

Pellicer Martínez, F., \& Martínez Paz, J. M. (2018). Climate change effects on the hydrology of the headwaters of the Tagus River: Implications for the management of the Tagus-Segura transfer. Hydrology and Earth System Sciences, 22(12), 6473-6491. https://doi.org/10.5194/hess-22$\underline{6473-2018}$

Peña-Gallardo, M., Gámiz-Fortis, S., Castro-Díez, Y., \& Esteban-Parra, M. (2016). Análisis comparativo de índices de sequía en Andalucía para el período 1901-2012. Cuadernos de Investigación Geográfica, 42(1), 67-88. https://doi.org/10.18172/cig.2946

Pita-López, M.F. (1989). La sequía como desastre natural. Su incidencia en el ámbito español. Norba: revista de geografía, 6,31-62.

Pita López, M. F. (2000). Un nouvel índice pour les domaines mediterranéens. Application au bassin du Guadalquivir (sud-ouest de IEspagne). Publications de IAssociation Internationale de Climatologie, 225-233. 
Portela, M. M., Zeleňáková, M., Santos, J. F., Purcz, P., Silva, A. T., \& Hlavatá, H. (2015). A comprehensive drought analysis in Slovakia using SPI. European Water, 51, 15-31. Retrieved from https://www.ewra.net/ew/pdf/EW_2015_51_02.pdf

Poquet, D., Belda, F., \& García-Haro, F. J. (2008). Seguimiento y regionalización de la sequía en la Península Ibérica mediante SPI y técnicas de teledetección. In Tecnologías de la Información Geográfica para el Desarrollo Territorial (pp. 707-713). Las Palmas de Gran Canaria: Servicio de Publicaciones y Difusión Científica de la ULPGC.

Pontes Filho, J. D., Portela, M. M., Marinho de Carvalho Studart, T., \& Souza Filho, F. de A. (2019). A Continuous Drought Probability Monitoring System, CDPMS, Based on Copulas. Water, 11(9), 1925. https://doi.org/10.3390/w11091925

Rajczak, J., Pall, P., \& Schär, C. (2013). Projections of extreme precipitation events in regional climate simulations for Europe and the Alpine Region. Journal of Geophysical Research: Atmospheres, 118(9), 3610-3626. https://doi.org/10.1002/jgrd.50297

Raziei, T., Martins, D. S., Bordi, I., Santos, J. F., Portela, M. M., Pereira, L. S., \& Sutera, A. (2015). SPI Modes of Drought Spatial and Temporal Variability in Portugal: Comparing Observations, PTO2 and GCPC Gridded Datasets. Water Resources Management, 29(2), 487504. https://doi.org/10.1007/s11269-014-0690-3

Ríos Cornejo, D., Penas Merino, Á., \& del Río González, S. (2013). Comparative analysis of precipitation trends in continental Spain over the period 1961-2010. International Journal of Geobotanical Research, 3(1), 1-18. https://doi.org/10.5616/ijgr130001

Richman, M. B. (1986). Rotation of principal components. Journal of climatology, 6(3), 293-335.

Rodrigo, F. S., \& Trigo, R. M. (2007). Trends in daily rainfall in the Iberian Peninsula from 1951 to 2002. International Journal of Climatology, 27(4), 513-529. https://doi.org/10.1002/joc. 1409

Santos, J. F., Pulido-Calvo, I., \& Portela, M. M. (2010). Spatial and temporal variability of droughts in Portugal. Water Resources Research, 46(3).

https://doi.org/10.1029/2009WR008071

Serrano Pérez, A., Mateos, V.L., \& García, J. A. (1999). Trend analysis of monthly precipitation over the iberian peninsula for the period 1921-1995. Physic and Chemistry of the Earth Part, 24(12), 85-90. https://doi.org/10.1016/S1464-1909 (98) 00016-1 
Serrano Notivoli, R. (2017). Reconstrucción climática instrumental de la precipitación diaria en España: ensayo metodológico y aplicaciones (Doctoral dissertation, Universidad de Zaragoza, Spain).

Serrano-Notivoli, R., De Luis, M., \& Beguería, S. (2017). An R package for daily precipitation climate series reconstruction. Enviromental Modelling \& Sofware, 89, 190195. http://dx.doi.org/10.1016/j.envsoft.2016.11.005

Serrano-Notivoli, R., Martín-Vide, J., Saz, M. A., Longares, L. A., Beguería, S., Sarricolea, P., Meseguer, O., \& de Luis, M. (2018a). Spatio-temporal variability of daily precipitation concentration in Spain based on a high-resolution gridded data set. International Journal of Climatology, 38, 518-530. https://doi.org/10.1002/joc.5387

Serrano-Notivoli, R., Beguería, S., Saz, M. Á., \& de Luis, M. (2018b). Recent trends reveal decreasing intensity of daily precipitation in Spain. International Journal of Climatology, 38(11), 4211-4224. https://doi.org/10.1002/joc.5562

Silva, A. T., Portela, M. M., \& Naghettini, M. (2012). Hydrology and Earth System Sciences Nonstationarities in the occurrence rates of flood events in Portuguese watersheds. Hydrology and Earth System Sciences, 16, 241-254. https://doi.org/10.5194/hess-16-241-2012

Silverman, B.W. (1986). Density Estimation for Statistics and Data Analysis. Vol 26, CRC Press.

Sousa, P. M., Trigo, R. M., Aizpurua, P., Nieto R., Gimeno L., \& Garcia-Herrera, R. (2011). Trends and extremes of drought indices throughout the 20th century in the Mediterranean. Natural Hazards Earth System Sciences, 11, 11-33. http://dx.doi.org/10.5194/nhess-11-33-2011 Spinoni, J., Naumann, G., Vogt, J. V., \& Barbosa, P. (2015): The biggest drought events in Europe from 1950 to 2012. Journal of Hydrology: Regional Studies, 3, 509524. https://doi.org/10.1016/j.ejrh.2015.01.001

Spinoni, J., Vogt, J. V., Naumann, G., Barbosa, P., \& Dosio, A. (2018). Will drought events become more frequent and severe in Europe? International Journal of Climatology, 38(4), 17181736. https://doi.org/10.1002/joc. 5291

Stagge, J. L., Tallaksen, L., \& Rizzi, J. (2015). Future meteorological drought: projections of regional climate models for Europe. In EGU General Assembly 2015. Viena, April 12-17. Retrieved from https://ui.adsabs.harvard.edu/abs/2015EGUGA..17.7749S/abstract

Szalai, S., \& Szinell, C. (2000). Comparison of Two Drought Indices for Drought Monitoring in Hungary - A Case Study. In J. Vogt \& F. Somma (Eds.), Drought and drought mitigation in 
Europe (pp. 161-166). Dordrecht: Kluver Academic Publishers. https://doi.org/10.1007/978$\underline{94-015-9472-1 \_12}$

Tallaksen, L. M., \& Lanen, H. A. J. van. (2004). Hydrological drought: processes and estimation methods for streamflow and groundwater. Amsterdam: Elsevier Science Limited.

Thiessen, A. H. (1911). Precipitation averages for large areas. Monthly weather review, 39(7), 1082-1089.

Turco, M., Ceglar, A., Prodhomme, C., Soret, A., Toreti, A., \& Doblas-Reyes F. J. (2017). Summer drought predictability over Europe: Empirical versus dynamical forecasts. Environmental Research Letters, 12(8). https://doi.org/10.1088/1748-9326/aa7859.

Vargas, J., \& Paneque, P. (2019). Challenges for the integration of water resource and drought risk management in Spain. Sustainability, 11(2), 308. https://doi.org/10.3390/su11020308

Vázquez López, L. A. (1999): Variabilidad interanual de la circulación atmosférica y escasez de precipitaciones en la península ibérica durante el invierno. In J. M. Raso Nadal \& J. Martín Vide (Eds.), La climatología española en los albores del siglo XXI (pp. 557-562). Barcelona: Asociación Española de Climatología.

Vicente Serrano, S.M., González-Hidalgo, J.C, De Luis, M., \& Reventós, J. (2004). Drought patterns in the Mediterranean area: The Valencia region (eastern Spain). Climate Research, 26, 515. https://doi.org/10.3354/cr026005

Vicente Serrano, S.M., Beguería Portugués, S., \& López Moreno, J.I. (2010). A multiscalar drought index sensitive to global warming: the standardized precipitation evapotranspiration index. Journal of climate, 23(7), 1696-1718. https://doi.org/10.1175/2009JCLI2909.1

Vicente-Serrano, S. M., Beguería, S., Lorenzo- Lacruz, J., Camarero, J. J., López-Moreno, J. I., Azorín Molina, C., Revuelto, J., Morán Tejeda, E., \& Sánchez-Lorenzo, A. (2012). Análisis comparativo de diferentes índices de sequía para aplicaciones ecológicas, agrícolas e hidrológicas. In Cambio Climático: extremos e impactos (pp. 679-689). Salamanca: Actas del VIII Congreso Internacional de la Asociación Española de Climatología.

Vicente-Serrano, S. M., Tomas-Burguera, M., Beguería, S., Reig, F., Latorre, B., Peña-Gallardo, M. Luna, Y., Morata, A., \& González-Hidalgo, J. C. (2017). A High-Resolution Dataset of Drought Indices for Spain. Data, 2(3), 22. https://doi.org/10.3390/data2030022

Wilhite, D.A., \& Glantz, M. H. (1985). Understanding the drought phenomenon: the role of definitions. Water International, 10, 111-120. 